\title{
Medullary Thyroid Carcinoma: An Update on Imaging
}

\author{
Sergiy V. Kushchayev $\mathbb{D}^{1,2}$, Yevgeniya S. Kushchayeva, ${ }^{3}$ Sri Harsha Tella ${ }^{1},{ }^{4}$ \\ Tetiana Glushko, ${ }^{5}$ Karel Pacak, ${ }^{6}$ and Oleg M. Teytelboym ${ }^{5}$
}

${ }^{1}$ Moffitt Cancer Center, Department of Radiology, 12902 USF Magnolia Drive, Tampa, FL 33612, USA

${ }^{2}$ Johns Hopkins Hospital, Department of Radiology, 601 N Caroline St, Baltimore, MD 21287, USA

${ }^{3}$ Diabetes, Endocrinology, and Obesity Branch, National Institute of Diabetes and Digestive and Kidney Diseases, National Institutes of Health, Bethesda 10, Center Dr, Bethesda, MD 20892, USA

${ }^{4}$ Department of Medicine, Division of Endocrinology, Diabetes and Metabolism, University of South Carolina School of Medicine, 6311 Garners Ferry Rd, Columbia, SC, USA

${ }^{5}$ Department of Radiology, Mercy Catholic Medical Center, 1500 Lansdowne Ave, Darby, PA 19023, USA

${ }^{6}$ Eunice Kennedy Shriver National Institute of Child Health \& Human Development (NICHD/NIH), 10 Center Dr, Bethesda, MD 20892, USA

Correspondence should be addressed to Sergiy V. Kushchayev; kushchayev@gmail.com

Sergiy V. Kushchayev and Yevgeniya S. Kushchayeva contributed equally to this work.

Received 7 April 2019; Accepted 8 May 2019; Published 7 July 2019

Academic Editor: Noriyuki Koibuchi

Copyright (C) 2019 Sergiy V. Kushchayev et al. This is an open access article distributed under the Creative Commons Attribution License, which permits unrestricted use, distribution, and reproduction in any medium, provided the original work is properly cited.

Medullary thyroid carcinoma (MTC), arising from the parafollicular C cells of the thyroid, accounts for 1-2\% of thyroid cancers. MTC is frequently aggressive and metastasizes to cervical and mediastinal lymph nodes, lungs, liver, and bones. Although a number of new imaging modalities for directing the management of oncologic patients evolved over the last two decades, the clinical application of these novel techniques is limited in MTC. In this article, we review the biology and molecular aspects of MTC as an important background for the use of current imaging modalities and approaches for this tumor. We discuss the modern and currently available imaging techniques-advanced magnetic resonance imaging (MRI)-based techniques such as whole-body MRI, dynamic contrast-enhanced (DCE) technique, diffusion-weighted imaging (DWI), positron emission tomography/computed tomography (PET/CT) with 18F-FDOPA and 18F-FDG, and integrated positron emission tomography/magnetic resonance (PET/MR) hybrid imaging - for primary as well as metastatic MTC tumor, including its metastatic spread to lymph nodes and the most common sites of distant metastases: lungs, liver, and bones.

\section{Introduction}

Thyroid neuroendocrine cells were first described in 1876 by Baber, and they were named $\mathrm{C}$ cells $(\mathrm{CC})$ due to the secretion of calcitonin (CTN) by Pearse in 1966 [1]. The human thyroid gland contains less than $0.01-0.1 \%$ CTN-producing parafollicular C cells. Medullary thyroid carcinoma (MTC) arises from these cells and accounts for 1-2\% of thyroid cancers. Although the majority of MTCs are sporadic, 25\% of cases are hereditary and are found in multiple endocrine neoplasia (MEN) 2A or 2B syndromes, or as part of familial MTC based on a specific germline mutation in the RET protooncogene [2].
MTC is frequently aggressive; on initial presentation only about half of patients (48\%) have localized disease, while $35 \%$ have a tumor extending beyond the thyroid into the surrounding tissues or regional lymph node metastases, and $13 \%$ have distant metastases typically to the lung, liver, or bones $[3,4]$. There is still no single optimal diagnostic imaging method to reveal all MTC recurrences or metastases, and therefore concurrent use of several imaging modalities is often required to provide the information needed for directing the management.

New functional techniques-such as dynamic contrastenhanced (DCE) magnetic resonance imaging (MRI), 


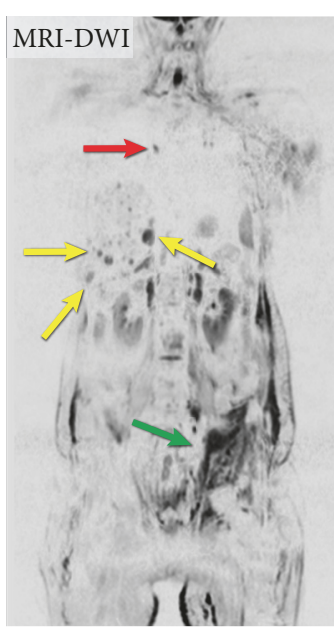

(a)

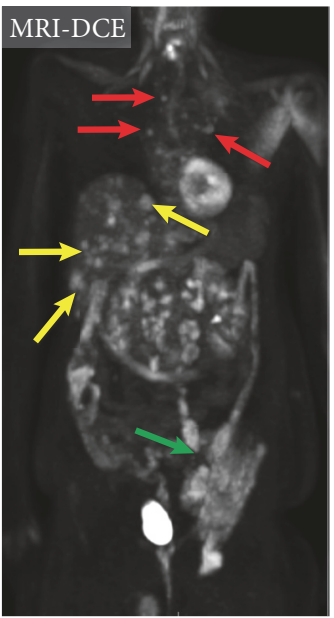

(b)

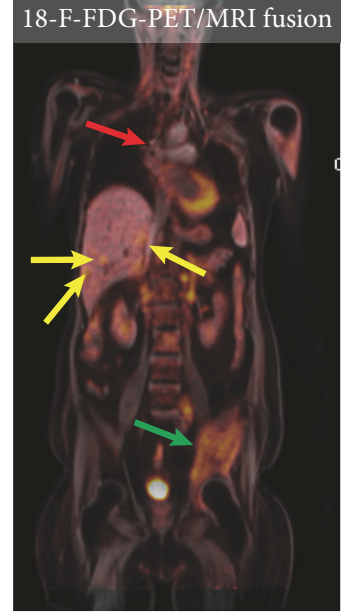

(c)

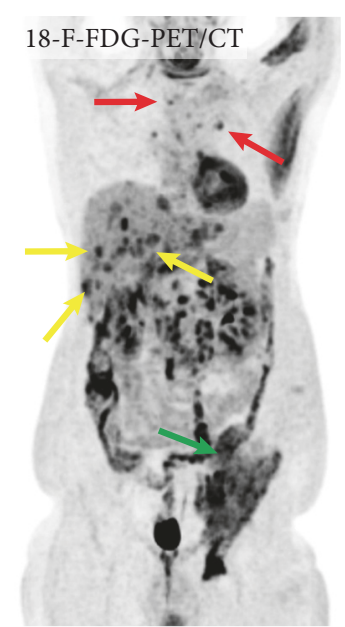

(d)

FIGURE 1: Imaging of metastatic medullary thyroid carcinoma using different radiological techniques: (a) whole-body magnetic resonance imaging, diffusion-weighted sequence (DWI), (b) whole-body contrast-enhanced magnetic resonance imaging, T1 weighted images (3D rendering DCE); (c) positron emission tomography/magnetic resonance imaging (18F-FDG-PET/MR imaging fusion technique); (d) positron emission tomography/computed tomography with 18F-FDG (18F-FDG PET/CT). Please note the excellent visualization of the multiple liver metastases (yellow arrow), mediastinal metastases (red arrow), and a large left iliac bone metastasis (green arrows) on DWI and DCE, comparable with 18F-FDG PET/CT, although they interrogate completely different biophysical tissue properties. Note that the images are not quite coregistered (arms up during PET acquisition and with arms down during MR imaging).

diffusion-weighted imaging (DWI) MRI, and positron emission tomography/computed tomography (PET/CT) using 18-fluorodeoxyglucose (18F-FDG), fluorine-18-dihydroxyphenylalanine (18F-FDOPA), and somatostatin (SST) analogs labeled with $68 \mathrm{Ga}$-have been recently introduced for the evaluation of oncologic patients. However, clinical applications of these modalities in MTC are very limited. The main aim of this article is to summarize current and outline new potentially helpful radiologic techniques, which have not yet been tested for MTC, but were found to be beneficial for other (neuroendocrine) malignancies.

\section{Modern Imaging Modalities and Techniques}

2.1. Ultrasound. Ultrasound (US) remains the most commonly used and preferred imaging modality for the evaluation of the location and characteristics of neck masses and thyroid nodules. US can be helpful in the evaluation of regional nodal metastases in patients with proven MTC, assessment for suspected recurrent disease, and high-risk patients for occult MTC. Color and Power Doppler techniques are useful for the evaluation of the vascular pattern of cervical lymph nodes. Fine needle aspiration with assaying CTN in the washout fluid helps diagnose MTC preoperatively [5].

2.2. Computed Tomography. The primary use of CT in MTC is to detect and characterize cervical and mediastinal lymph nodes and evaluate lung parenchyma for metastases. For patients with suspected hepatic metastases, multiphase CT scanning includes nonenhanced, late arterial phase, portal venous phase, and delayed phase imaging; however, it is less effective than MRI techniques. Nonenhanced CT provides characterization of bony changes secondary to metastases; however, the evaluation of the bone marrow infiltration, spinal canal, and soft tissue is limited and requires further imaging with MRI.

2.3. Magnetic Resonance Imaging. MRI provides excellent visualization of metastatic lesions in soft tissues, intraabdominal lymph nodes, bones, and liver and can help evaluate the extent of the disease during the initial staging and assess the treatment response. Several new MRI techniques in oncologic imaging-including whole-body MRI (WBMRI), DCE technique, DWI, and integrated PET/MR hybrid imaging-not only allow the evaluation of the biological and functional effects of treatment, but also offer the opportunity to evaluate treatment response, study tumor pathophysiology, and heterogeneity and may also predict clinical outcomes, particularly in the setting of novel adjuvant therapies [6] (Figure 1).

$W B-M R I$ is a potentially useful technique for the initial staging and evaluation of treatment response. The technique is particularly useful in children and young adults because it does not use ionizing radiation [7].

DWI measures the diffusion of water molecules (Brownian movement) within a given tissue, which is mainly dependent on cellular density. Tissues with a high cellularity_including malignancies-have a smaller extracellular space, resulting in restricted water molecule diffusion and a high DWI signal. The degree of proton diffusion restriction can be quantified via the apparent diffusion coefficient (ADC) [8]. Numerous studies have shown that $\mathrm{ADC}$ values in a number of nonthyroid tumors 
can be used to access treatment response [9]. WB scanning utilizing DWI sequence (WB-DWI) has recently been introduced as an effective evaluation of metastases burden in oncology (Figure 1(a)). WB-DWI is not a replacement for PET/CT for the evaluation of oncological patients; indeed, these techniques are complementary because they interrogate completely different biophysical tissue properties (glucose metabolism vs. cellular density) [10]. WB-DWI might be of value in recognizing non-FDG-avid metastases and evaluating anatomic regions with high physiologic radiotracer uptake. However, at this point there are no studies that have evaluated the utility of DWI techniques for patients with metastatic MTC.

$D C E-M R I$ is being used in oncology to measure properties of tumor microvascular structure and permeability. DCE-MRI becomes an attractive modality for evaluating antiangiogenic cancer therapies, assessing changes in tumor vasculature, and predicting tumor shrinkage [11]. This technique may have a clinical application in MTC, as targeting angiogenesis produced the most impressive clinical treatment responses for metastatic MTC to date [12]. Only one study reported the exploratory use of DCE-MRI in 10 patients in order to evaluate tumor perfusion as a surrogate of the antiangiogenic activity of sorafenib [13]. Since capillary perfusion determines the delivery of drugs to tumor cells, recent studies have used the in vivo measurement of capillary perfusion by DCE-MRI to monitor perfusion changes in response to antiangiogenic agents [8] (Figure 1(b)).

$P E T / M R$ hybrid imaging is an innovative technique still under investigation. MR imaging can depict metastatic bone marrow involvement before the development of structural damage that is visible on a CT scan. A large meta-analysis based on 57 publications showed that the diagnostic performance of simultaneous PET/MR imaging was similar or even better than PET/CT for most anatomical locations and most oncological diseases; however, PET/CT was superior for small lung nodule detection [14] (Figure 1(c)).

2.4. Nuclear Medicine Techniques. There are numerous conventional (such as a bone scan or metaiodobenzylguanidine (MIBG) scintigraphy) and advanced (PET/CT scans with different radiopharmaceuticals) nuclear medicine techniques that can be useful for patients with MTC. There is no general agreement on their routine use in MTC $[15,16]$ (Figure 2).

Several studies have evaluated the diagnostic accuracy of different radiotracers for PET/CT in patients with suspected recurrent MTC. The majority of these studies were limited by the small number of patients, as the disease is rare. Some of the studies showed conflicting results. In 2012 Treglia et al. published two large meta-analyses evaluating the diagnostic accuracy of PET/ CT with 18F-FDOPA and 18F-FDG [17, 18]

2.4.1. 18F-FDOPA. 18F-FDOPA PET/CT represents the single best modality for whole-body MTC metastasis detection [19]. 18F-FDOPA PET/CT is particularly suitable for the detection of small metastatic lymph nodes (around $6 \mathrm{~mm}$ ) [20]. Moreover, 18F-FDOPA PET/CT is a sensitive technique to identify liver metastases not detected by $18 \mathrm{~F}-\mathrm{FDG}$ as well as MTC metastases in unusual locations [20]. In patients with rising tumor markers, detection rates are considerably higher than with other imaging methods (66 \% patient-based, $71 \%$ lesion based), which is of clinical significance since imaging with 18F-FDOPA is often performed in patients who have negative results on conventional imaging and/or 18F-FDG PET/CT [18]. Using the proposed CTN cutoff for additional imaging according to the American Thyroid Association (ATA) guidelines [15], the sensitivity of 18F-FDOPA PET for metastatic/recurrent MTC is $79-100 \%[19,21]$. In the metaanalysis by Treglia et al., the sensitivity of 18F-FDOPA PET or PET/CT for metastatic/recurrent MTC was $73 \%$ if CTN values were above $150 \mathrm{pg} / \mathrm{ml}$ (551 pmol/l) [18].

18F-FDOPA PET is superior to 18F-FDG PET in the evaluation of metastatic/recurrent MTC, with a higher patient-based sensitivity ( $64 \%$ vs. $48 \%$, respectively; range, $38-83 \%$ vs. $17-64 \%)$ and lesion-based sensitivity ( $72 \%$ vs. $52 \%$, respectively; range, $52-94 \%$ vs. $28-62 \%$ ) [22]. The sensitivity increases when both imaging modalities are used [22]. The patient-based detection rate increases significantly with CTN levels $>150 \mathrm{pg} / \mathrm{ml}(551 \mathrm{pmol} / \mathrm{l})$ vs. CTN $<150 \mathrm{pg} / \mathrm{ml}$ (551 pmol/l) for both 18F-FDOPA PET/CT (sensitivity $91 \%$ vs. $29 \%$ ) and $18 \mathrm{~F}-\mathrm{FDG}$ PET/CT (sensitivity $73 \%$ vs. $14 \%$ ) [20]. The diagnostic accuracy of 18F-FDOPA and 18F-FDG $\mathrm{PET} / \mathrm{CT}$ increases significantly when a carcinoembryonic antigen (CEA) cutoff of $>5 \mathrm{ng} / \mathrm{mL}$ is used (sensitivity of $81 \%$ and $73 \%$, respectively, vs. $43 \%$ for $18 \mathrm{~F}-\mathrm{FDOPA}$ PET/CT and $14 \%$ for 18 F-FDG PET/CT when CEA $<5 \mathrm{ng} / \mathrm{mL}$ ) [20]. In a large meta-analysis, Treglia et al. also found higher detection rates of 18F-FDOPA PET or PET/CT when setting the cutoff of CEA at $5 \mathrm{ng} / \mathrm{mL}$ (detection rate of $64 \%$ with CEA > 5 $\mathrm{ng} / \mathrm{mL}$ vs. $48 \%$ with CEA $\leq 5 \mathrm{ng} / \mathrm{mL}$ ) [18].

2.4.2. 18F-FDG PET/CT. 18F-FDG accumulates in neoplastic cells using glucose as an energy source mainly according to their proliferative activity. However, neuroendocrine tumors (NETs), including MTC, frequently show an indolent course and, consequently, a low 18F-FDG uptake [23]. A detection rate of $18 \mathrm{~F}-\mathrm{FDG} \mathrm{PET} / \mathrm{CT}$ on a per patient-based analysis ranged from 24 to $95 \%$ with a pooled estimate of 59\% (95\% CI: 54-63\%) and improved in patients with increased serum CTN and CEA levels, and shorter serum CTN and CEA doubling times $[4,17,18,24]$. The lowest CTN levels with positive 18F-FDG PET have a wide range in the literature from 129 to $816 \mathrm{pg} / \mathrm{ml}$ (473-2996 pmol/l) [25-27]. 18F-FDG PET rarely detects disease in patients with CTN levels below $500 \mathrm{pg} / \mathrm{ml}$ (1836 pmol/l) [26]. Sensitivity was $78 \%$ and $20 \%$ for CTN above 1000 (3671 pmol/l) and below 1000 pg/ml (3671 pmol/l), respectively [26]. The average CTN level in patients with a positive 18F-FDG PET was found to be $2311 \mathrm{pg} / \mathrm{ml}$ (8483 pmol/l) with a range of 51-247,000 pg/ml (187-906737 $\mathrm{pmol} / \mathrm{l})$ in one study [27] and $7260 \mathrm{pg} / \mathrm{mL}$ (26651 pmol/l) with a range of $106-541,000 \mathrm{pg} / \mathrm{ml}(389-198,6011 \mathrm{pmol} / \mathrm{l})$ in another [26]. However, a negative 18F-FDG PET has been described even with a CTN of 55,200 pg/ml (202,639 pmol/l) [26]. The maximum standardized uptake value (SUV max) has been shown to correlate with CTN levels [24, 28]. 18FFDG PET/CT should not be considered as first-line diagnostic imaging methods in patients with suspected recurrent MTC but could be very helpful in detecting recurrence in 


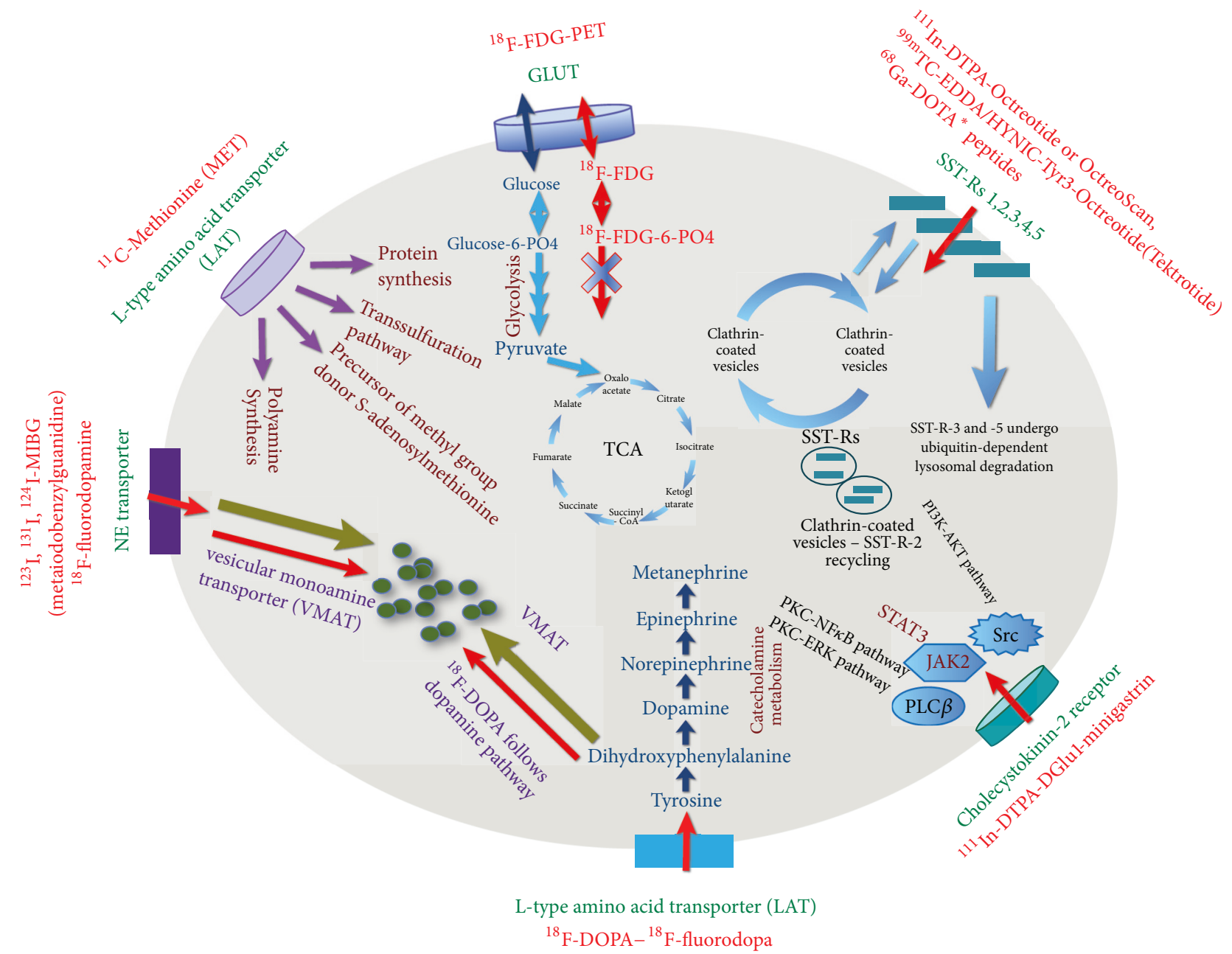

Figure 2: Mechanisms of uptake and localization of different radiopharmaceuticals, which can be used for medullary thyroid carcinoma. Please see explanations in the text.

those patients in whom a more aggressive disease is suspected $[4,29]$.

2.4.3. Somatostatin Receptor Scintigraphy. Somatostatin (SST) is a regulatory peptide widely distributed in the human body that binds to somatostatin receptors (SST-R types 1-5). SST-R expression can be visualized using conventional scintigraphy or PET/CT with a number of different tracers. 111In-octreotide has high affinity for SST-R-2, but poor nuclear imaging properties with detection rates between 20 and 64\% [30]. Newer radiolabeled SST-R analogs have been developed. Using 1,4,7,10-tetraazacyclododecane1,4,7,10-tetraacetic acid (DOTA) as a universal chelator that provides stable complexes with $111 \mathrm{In}, 68 \mathrm{Ga}, 177 \mathrm{Lu}$, and $90 \mathrm{Y}$ and is used to label SST-R analogs maintains biological activity that is sufficient for imaging and therapy. 68GaDOTA, 68Ga-DOTANOC (SST-R-2,3,5), 68Ga-DOTATATE (SST-R-2), 68Ga-DOTATOC (SST-R-2 and 5), 68Ga-DOTANOC (SST-R-2,3,4 and 5), and 99mTc-EDDA/HYNIC-Tyr3Octreotide (Tektrotide) have important clinical implications.
PET/CT with 68Ga-labeled SST-R compounds is a valuable diagnostic tool for patients with NETs. Experience in metastatic and recurrent MTC is limited compared to $18 \mathrm{~F}$ FDG and 18F-DOPA PET/CT with inferior results compared with the other two tracers as MTC has lower density and an inhomogeneous expression of SST-R compared to other NETs [31]. 68Ga-DOTATATE PET/CT is superior to 111Inoctreotide SPECT/CT for the detection of recurrent MTC demonstrating a significantly higher number of lesions [31]. As is the case with 18F-FDG and 18F-FDOPA PET/CT, the detection rate of SST-R analogs PET/CT in recurrent MTC increases in patients with higher serum CTN levels [23]. Nevertheless, SST-R analogs PET/CT may have an additional role, as this method could be useful in the selection of patients with inoperable tumors for peptide receptor radionuclide therapy with 90Y-DOTA0-Tyr3-octreotide (90Y-DOTATOC) or 177Lu-DOTA0-Tyr3-octreotate (177Lu-DOTA-TATE) [30].

A meta-analysis of nine studies devoted to the diagnostic performance of SST-R analogs PET or PET/CT (including 68Ga-DOTATATE, 68Ga-DOTANOC, 68Ga-DOTANOC, 
and 68Ga-DOTALAN) in patients with recurrent MTC demonstrated a suboptimal detection rate of $64 \%$ (a per patient-based analysis), which increases to $83 \%$ for CTN $>500 \mathrm{ng} / \mathrm{L}$ [32]. Overall, the diagnostic performance of SST$\mathrm{R}$ analogs PET or PET/CT in recurrent MTC is overall lower compared to 18F-FDOPA PET/CT [32]. Although 68GaDOTATATE PET/CT is not an optimal whole-body imaging technique as a single imaging modality in patients with MTC, it detects $100 \%$ for bone metastases and it was found to be superior to bone scan that identified $44 \%$ of osseous lesions [33].

\subsubsection{Cholecystokinin Receptor Subtype 2 (CCK2R)/Gastrin} Receptors and Gastrin Receptor Scintigraphy. The cholecystokinin receptor subtype 2 is an important promoter of tumor growth. It has been shown to be expressed in normal C cells and overexpressed in numerous NETs, including MTC $[34,35]$. Blaker et al., based on an analysis of 19 MTC patients, found that CCK2Rs could be detected in almost all early-stage tumors, such as T1 and T2 stages, while further growth and potential loss of cell differentiation, i.e., in T3 and T4 primary and metastatic tumors, might be associated with a loss of CCK2Rs [35]. Two DOTA-minigastrin analogs radiolabeled with 111In and $68 \mathrm{Ga}$ were evaluated in a preclinical in vivo model and showed higher uptake than $68 \mathrm{Ga}$-labeled cyclic DOTA-peptides [36]. The injection of radiolabeled minigastrin was associated with mild side effects such as nausea, flushing, and hypotension that are similar to pentagastrin testing [37]. Since there is no physiologic uptake in liver and spleen, 111In-DTPA-DGlul-minigastrin favors the detection of metastatic lesions in these organs [38]. The combination of CT with a 111In-DTPA-DGlul-mini-gastric scan resulted in the detection of $96.7 \%$ of known lesions [37]. However, it was not accurate for occult disease [37]. The main limitations of this technique are the limited availability and that singlephoton emission computed tomography (SPECT-CT) scan does not provide sufficient spatial resolution for the precise detection of lesions and high-precision surgical planning.

2.4.5. MIBG. Both 131I-MIBG and 123I-MIBG are available for diagnostic purposes. With a cumulative sensitivity for MTC between 30 and 50\% [39, 40], they are not recommended except for imaging of pheochromocytomas in case of MEN2, a syndrome where MTC is found.

2.4.6. 11C-Methionine. Methionine is an essential amino acid necessary for protein synthesis. However, this tracer has a considerable nonprotein metabolism and generates substantial amounts of nonprotein metabolites, making the correct quantification of protein synthesis difficult [41]. 11CMethionine was more sensitive to the detection of cervical lymph nodes when compared to 18F-FDG, but not better than neck US or a combination of 18F-FDG PET with neck US for lymph node detection [25]. 11C-Methionine, as well as 18F-FDG, was more sensitive in cases of elevated CTN with a cutoff $>370 \mathrm{pg} / \mathrm{ml}(1358 \mathrm{pmol} / \mathrm{l})$; however, the high physiologic uptake of 11C-MET in the liver that limits visualization of of hepatic metastases [25].
2.4.7. Immunoimaging. Using directly labeled antibodies, their fragments, or antibody-derived recombinant constructs has been proposed for the radionuclide targeting of tumors. Immuno-PET using anti-CEA antibodies labeled with 111In, $131 \mathrm{I}$, or $68 \mathrm{Ga}$ was reported to be potentially accurate for detecting relapsing MTC [42]. Further studies are required to confirm the accuracy of this new technique [42].

2.4.8. Bone Scan. Although, the current 2015 ATA guidelines recommend bone scintigraphy for patients with extensive neck disease, regional or distant metastases, and a serum CTN level greater than $500 \mathrm{pg} / \mathrm{ml}(1836 \mathrm{pmol} / \mathrm{l})$, the diagnostic value of this technique for MTC is not well-investigated. As patients with suspected distant metastases due to MTC, including bone metastases, should undergo PET/CT or PET/MRI with 18F-FDG and 18F-FDOPA anyway, these techniques appear more efficient for the detection of bone lesions, rather than bone scintigraphy.

\section{Clinical Application of the Imaging Techniques}

3.1. Preoperative Evaluation. Most patients with sporadic MTC have unifocal disease and present with a palpable neck mass usually in the fifth or sixth decade of life. These tumors tend to be located in the posterior thyroid and therefore can compress or invade local structures, causing hoarseness, dysphagia, or respiratory difficulty [43]. Sonographic findings of primary MTC are often not specific. A large meta-analysis that included 157 malignancies showed that the majority (83\%) of tumors were hypoechoic, $38 \%$ had irregular margins, $36 \%$ demonstrated microcalcifications, and $27 \%$ showed macrocalcifications (Figure 3). Compared with papillary thyroid carcinoma, MTCs were found to be larger, and more frequently showed cystic changes and homogeneous echotexture of the solid portion [44]. The US appearance of MTC nodules was divided into two categories: m-MTC type with aggressive US features and b-MTC type without aggressive US features $[45,46]$. Metastatic spreading of the malignancy to lymph nodes, extrathyroidal invasion, moreadvanced TNM stage, and higher postoperative CTN levels were more frequent in the m-MTC group $[45,46]$. CT and MRI are generally used for the evaluation of larger thyroid nodules (greater than $3 \mathrm{~cm}$ in diameter) and to evaluate substernal extension and invasion into adjacent structures [47]. Sonographic patterns proposed by the 2015 ATA Thyroid Nodules and Differentiated Thyroid Cancer guidelines were primarily aimed at differentiated thyroid cancer, but these recommendations were also found to perform well in MTC [48].

Cytology evaluation alone is not enough for preoperative evaluation and to guide initial surgery [49]. Ultrasoundguide fine needle aspiration cytology (FNAC) of a thyroid nodule cannot always reliably distinguish between MTC and other thyroid neoplasms including adenomas. Sensitivity of FNAC was shown to be $63 \%$ vs. $98 \%$ for serum CTN measurement with only $74.5 \%$ cases diagnosed by FNAC in patients with elevated CTN level [50]. FNAC is a very important 


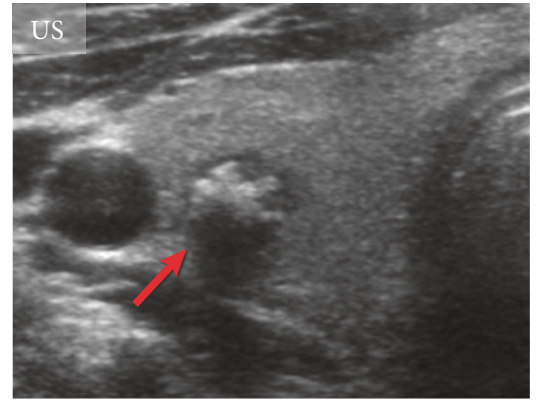

(a)

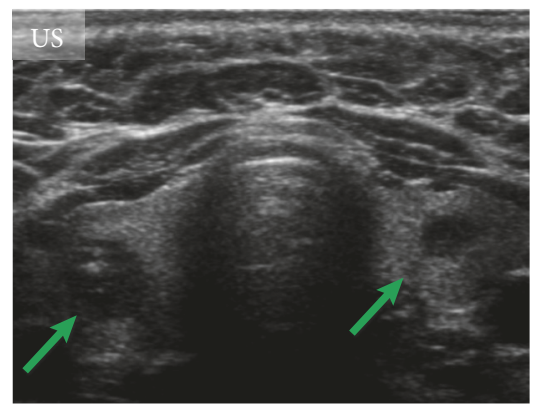

(d)

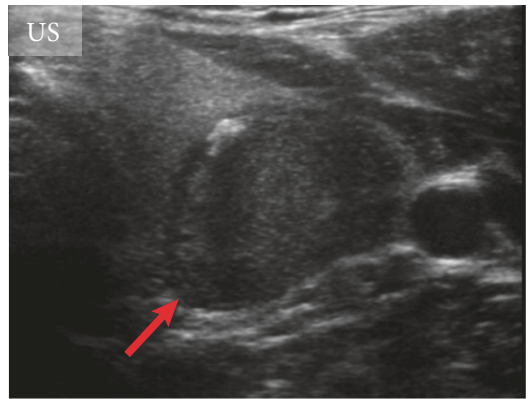

(b)

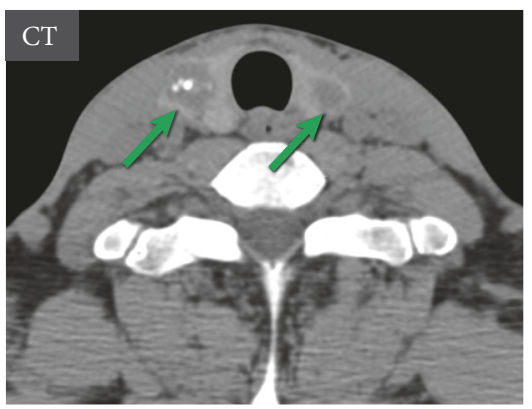

(e)

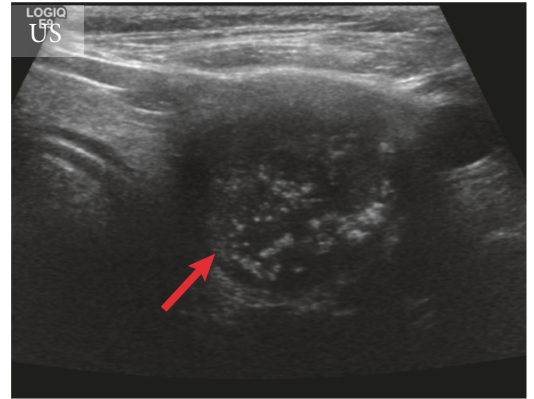

(c)

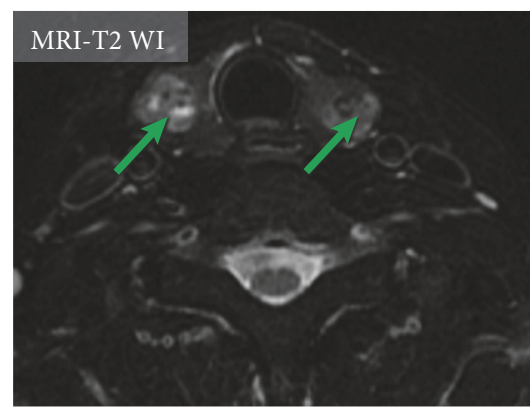

(f)

FIGURE 3: Imaging of the primary tumor in medullary thyroid carcinoma. (a)-(c) Ultrasound (US) features of primary medullary thyroid carcinoma of different patients (red arrows). US of the thyroid gland shows markedly hypoechoic nodules with marked calcifications. (d)(f) US of the neck, computed tomography, and magnetic resonance tomography (T2 weighted images of the neck) of the same patient with bilateral medullary thyroid carcinomas (green arrows).

measure in the preoperative workup of patients with thyroid nodules; however, it is controversial due to questions of efficacy, accuracy, and cost-effectiveness [51]. Serum CTN has also some limitations including high false positive results, low positive predictive value, and lack of agreement for CTN threshold to suspect MTC [51,52]. Diagnosis of MTC in thyroid nodules with undetermined cytology using MTC gene classifier was shown to demonstrate a high sensitivity of $97.9 \%$, specificity of $99.8 \%$, and positive and negative predictive values of $97.9 \%$ and $99.8 \%$, respectively [51].

If the preoperative diagnosis of MTC is missed and surgery starts with a diagnostic hemithyroidectomy, reoperation is needed to perform total thyroidectomy. A definitive diagnosis is often made only on surgical histopathology [53].

As surgery remains the only curative treatment for patients with MTC, the ultimate goal of preoperative evaluation is to identify all distant metastatic lesions and, most importantly, metastatic lymph nodes. Lymph node involvement is seen in $35-50 \%$ and distant metastases are present in $10-15 \%$ of patients at the time of diagnosis [2]. Different imagining modalities show distinct detection rates for lesions in different organs; therefore, radiological workup should be tailored based on the most suitable method for each organ (system).

Preoperative evaluation of the extent of the primary tumor and lymph node involvement with US is an essential procedure. In cases when the primary tumor is $1 \mathrm{~cm}$ or larger, the risk of lateral neck metastasis is higher, and careful US examination of the lateral neck nodes should be performed [54] (Figure 4). However, 36\% of patients might have false negative results on preoperative neck US, especially in central neck compartment [55]. Thirty-eight percent of patients with node-negative MTC were shown to fail normalization of CNT level after surgery despite extensive surgical treatment, which is suggestive of the possibility of distant metastases [56]. More intensive imaging, invasive diagnostic techniques, and extension of follow-up can likely improve the ascertainment rates of distant metastases [56]. Aggressive evaluation of the suspicious appearing neck lymph nodes should undergo FNAC with CTN assay of washout fluid. The current ATA guidelines recommend imaging procedures to exclude metastatic MTC for patients with extensive neck disease and signs/symptoms of regional or distant metastases and all patients with CTN $>500 \mathrm{pg} / \mathrm{ml}$ (1836 pmol/l) [15].

This recommendations is based on the study published by Machens and Dralle in 2010 [57]. Patients who underwent thyroidectomy and lymph node dissection and had preoperative CTN $<100 \mathrm{pg} / \mathrm{ml}$ (367 pmol/l) could be biochemically cured [57]. However, only $81 \%$ of patients with a CTN level of $100-500 \mathrm{pg} / \mathrm{ml}$ (367-1836 pmol/l) who underwent surgery had a normal postoperative CTN level [57]. Thus, every fifth patient with CTN levels of 100-500 pg/ml (367-1836 pmol/l) probably had metastatic disease, which could not be resected during the surgery or might have been located outside of the surgical area, while imaging findings were not analysed in this study. Moreover, preoperative CTN levels 


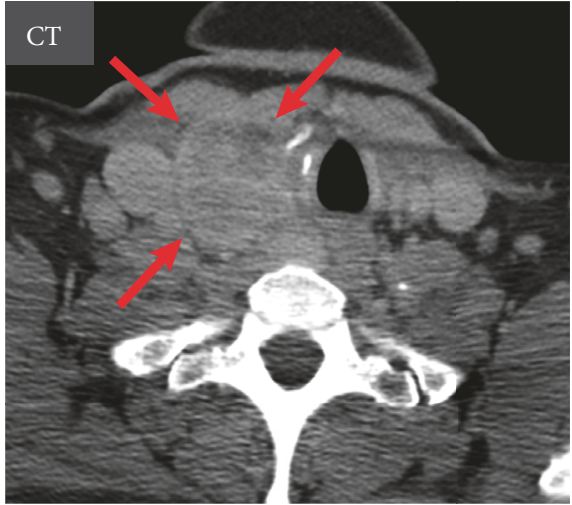

(a)

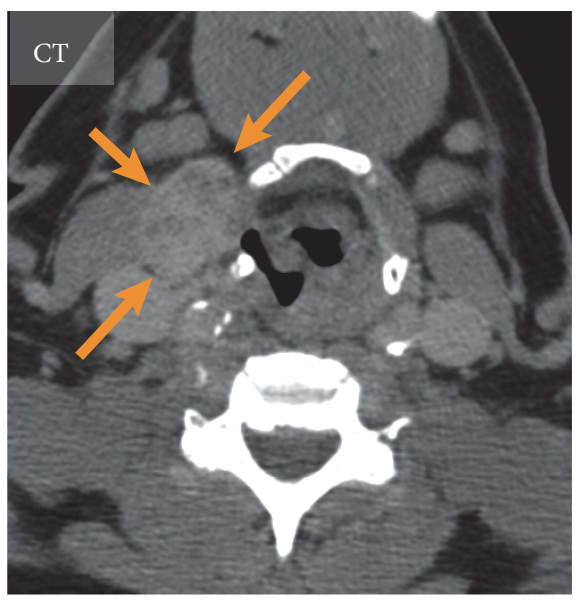

(c)

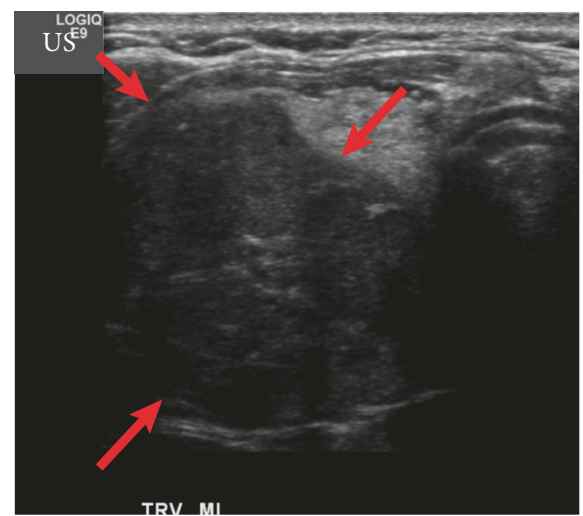

(b)

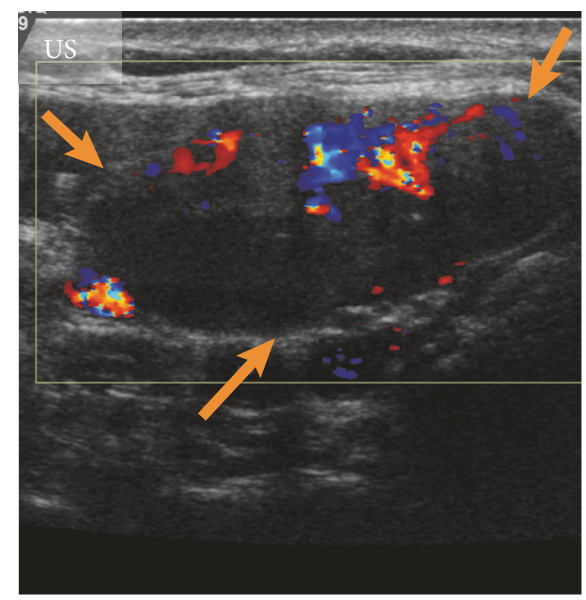

(d)

Figure 4: Imaging of the medullary thyroid carcinoma with metastasis to lymph node. Medullary thyroid carcinoma of the right thyroid lobe (red arrows) visualized on axial computed tomography (CT) (a) and transverse ultrasound (US) scan (b). A large metastatic lymph node (orange arrows) is demonstrated on axial CT of the neck (c) and sagittal US (d).

do not always reflect the actual stage of a patient's disease $[58,59]$. These levels correlate with the tumor mass and the secretory capacity of the malignant cells and, hence, they do depend on the degree of (de)differentiation [59]. While these aspects require further research, patients with preoperative CTN levels above $100 \mathrm{pg} / \mathrm{ml}$ (367 pmol/l) might benifit from comprehansive imaging and, idealy, from 18F-FDOPAPET/CT. This may also be informative for surgical planning (for example, a patient with mediastinal metastatic lymph nodes may require thoracotomy), plan other therapeutic strategies, and establish a "baseline" to assess response to treatments. The use of PET/CT for accurate initial staging and treatment planning is established in many malignancies [60] and can be potentially applied to MTC.

3.2. Postoperative Follow-Up Patients with MTC. Follow-up after surgical therapy for MTC typically starts 2-3 months postoperatively by obtaining new baseline CTN and CEA levels. Patients who have undetectable CTN levels postoperatively can be followed with measurements of serum CTN and CEA initially every 6 months for the first year and then annually [15].
In the elegant study of Pellegrini et al. (2003), the active follow-up of the MTC patients allowed the early diagnosis of the metastatic disease at basal serum CTN levels $<150 \mathrm{pg} / \mathrm{ml}(551 \mathrm{pmol} / \mathrm{l})$ with regional and/or distant metastatic lesions $[15,58]$. Among five patients with CTN level $<150 \mathrm{pg} / \mathrm{ml}$ at the moment of relapse, two patients had distant metastases, two patients had cervical disease, and one had regional and distant metastases. Of note, the imagingdetected relapses were diagnosed in patients who underwent treatment between 1988 and 1998, and available imaging workup included a yearly neck and abdominal US, chest CT without CT of abdomen, and a bone scintigraphy; no MRI or PET/CT was used [58]. In the cohort of 18 patients with imaging-detected relapses in three patients, postoperative CTN level was undetectable. Authors emphasized that even undetectable pentagastrin-stimulated CTN cannot exclude the risk of recurrence and, thus, MTC patients need a prolonged follow-up [58].

MTC includes genetically heterogeneous subtypes of malignancies that demonstrate different clinical courses from very aggressive to slowly progressing. Therefore, an individualized/differentiated approach for imaging and management 


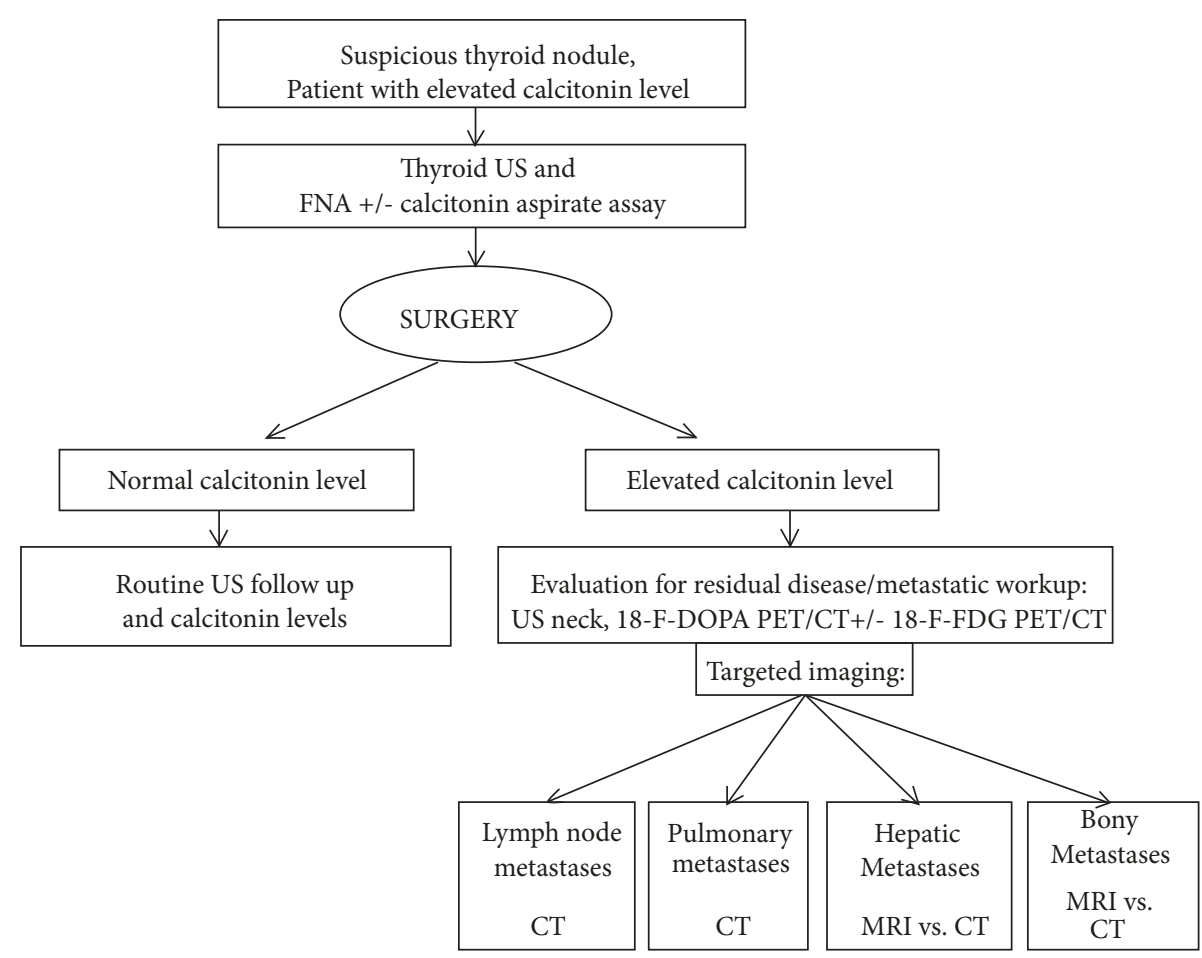

FIGURE 5: The proposed management approach for patients with medullary thyroid carcinoma. Please see explanations in the text.

of patients is optimal. However, currently, there are no meaningful data to offer clinical or histopathological criteria for different imaging protocols. It can be suggested that any patient with elevated postoperative CTN levels should be considered as having metastasis disease of unknown location and should promptly undergo comprehensive imaging: US, CT, or MRI of the neck, 18F-FDOPA PET/CT possibly with 18F-FDG PET/CT, and then targeted imaging depending on specific organ involvement (Figure 5). However, future studies that measure patient outcomes and cost-effectiveness are warranted.

In some patients, biological markers (CNT and CEA levels and doubling times) may not suffice for surveillance since they may not address the heterogeneity of response at different sites as therapy-resistant clones develop [61]. Given the availability of powerful radiological technologies, there is a need to perform comprehensive studies to evaluate correlations between biological markers and the location of the metastases in patients with advanced MTC in order to establish imaging protocols and diagnostic algorithms.

3.2.1. Lymph Nodes. US of the neck is the most accurate modality for the detection of recurrent disease in the neck [27]. US features that should arouse suspicion about lymph node metastases include a rounded bulging shape, increased size, replaced fatty hilum, irregular margins, heterogeneous echotexture, calcifications, cystic areas, and vascularity throughout the lymph node instead of normal central hilar vessels on Doppler imaging [62]. US is less accurate for detecting central compartment disease than in the lateral compartments, as up to $80 \%$ of patients will have metastases to the central neck nodes. One of the most common pitfalls in cervical US is not to examine the lateral neck. If US suspects metastatic lymph nodes, then contrast-enhanced CT of neck and chest (for the evaluation of mediastinum) is a reasonable next step in imaging. Suspicious enhancing or abnormally appearing neck lymph nodes should undergo fine needle aspiration (FNA) with CTN assay of washout fluid. The reliable identification of mediastinal lymph node metastases under $1 \mathrm{~cm}$ is challenging, as mediastinal lymph nodes under $1 \mathrm{~cm}$ are considered normal on CT examination, and FNA of these nodules is not feasible. Therefore, subsequent 18FFDOPA PET/CT may localize these small metastatic foci and provide guidance for surgeons.

3.2.2. Imaging of Distant Metastases. Currently, the further imaging for the detection of distant metastases is recommended when the postoperative serum CTN level is $>150 \mathrm{pg} / \mathrm{ml}(551 \mathrm{pmol} / \mathrm{l})$ [63]. Even though no step-by-step recommendations for patients with occult elevated CTN and CEA levels were suggested, the published studies have confirmed the superiority of 18F-FDOPA PET/CT over all other radiopharmaceuticals in the localization and detection of occult metastatic lesions [23, 29] (Figure 6). 18F-FDOPA $\mathrm{PET} / \mathrm{CT}$ demonstrated the highest sensitivity in the detection of the metastatic lymph nodes compared to $18 \mathrm{~F}-\mathrm{FDG}$ and 68Ga-DOTATATE PET/CT [23]. 18F-FDOPA retention is a feature of high differentiation, while the accumulation of 18F-FDG reflects poor differentiation of the MTC cells [23]. For the imaging of recurrent disease, the two tracers act in a complementary manner to provide the maximum benefit from their combined sensitivity [23] (Figure 7). 18F-FDOPA 


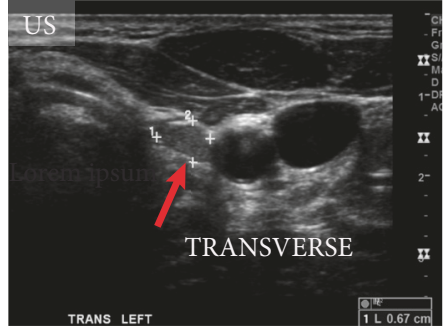

(a)

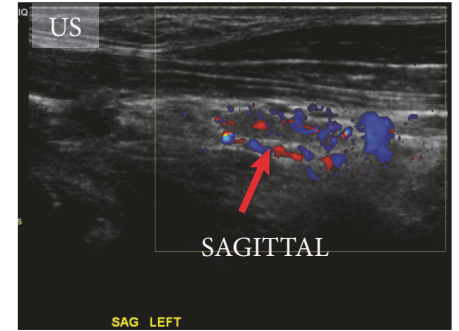

(b)

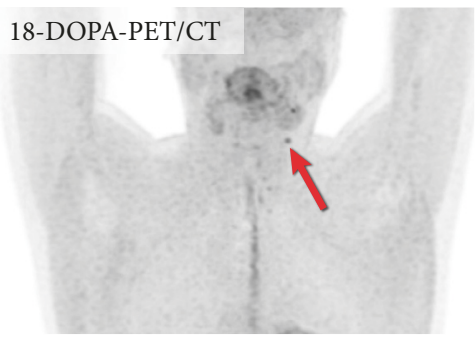

(c)

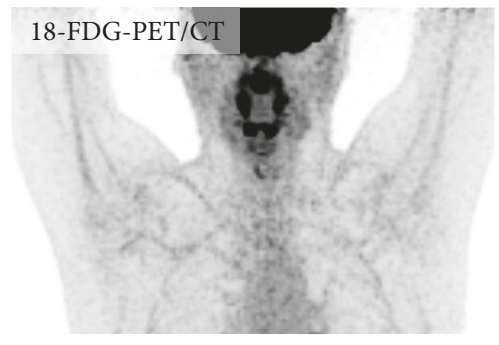

(d)

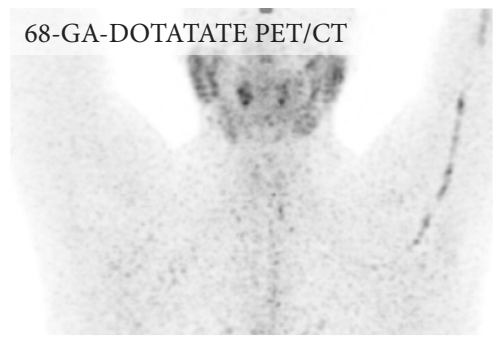

(e)

FiguRE 6: Mapping of the metastatic lymph nodes in medullary thyroid carcinoma. Asymptomatic patient after thyroidectomy for medullary thyroid carcinoma and elevated calcitonin level $(54 \mathrm{pg} / \mathrm{ml})$ was evaluated for possible neck lymph node metastases. Ultrasound of the neck showed several suspicious lymph nodes (not shown). One of the lymph nodes (b) was found to be positive on only 18F-FDOPA-PET/CT (c), while $18 \mathrm{~F}-\mathrm{FDG}-\mathrm{PET} / \mathrm{CT}$ and $68 \mathrm{Ga}$-DOTATATE-PET/CT were negative.

still remains an 18-F-FDOPA is still not FDA approved diagnostic radiopharmaceutical for MTC in the USA and is approved for clinical use in some European countries. Unfortunately, not all institutions have 18F-FDOPA available; therefore, in many cases patients need to be referred to specialized centers in the US and Europe.

For distant metastases, nuclear medicine imaging with different radiopharmaceuticals is considered a type of "screening technique": when the metastatic involvement of the particular organ is suspected or identified, a targeted evaluation of the organ with cross-sectional imaging is needed.

(1) Lung Metastases. Lung metastases occur in 33\% of patients with MTC [27]. They are typically numerous and may be associated with mediastinal lymph node metastases [63]. Pulmonary metastases in MTC may exhibit a micronodular pattern or have a large, well-circumscribed, round macronodular appearance, also known as a "cannonball" pattern. Micronodular metastases have a nonspecific appearance, which may calcify and can be easily mistaken for granulomatous diseases, such as tuberculosis, histoplasmosis, or sarcoidosis [64, 65]. A lymphangitic spread of the tumor with amyloid deposition in peribronchovascular structures without alveolar involvement resulting in reticulonodular perihilar opacity on imaging was also reported [66]. Chest radiograph is a insensitive technique to diagnose lung metastases; about half of pulmonary lesions $<5 \mathrm{~mm}$ remain undetected [67]. CT is excellent at visualizing pulmonary metastases; they appear as well-circumscribed round soft tissue lesions, most often in the lower lungs. MR imaging is not commonly used for follow-up diagnosis or evaluations of pulmonary metastases. Pulmonary lesions larger than $5 \mathrm{~mm}$ may be readily identified on MR imaging; however, smaller nodules are detected with less sensitivity [68]. Therefore, MR imaging cannot replace CT for the diagnosis of pulmonary metastases. DWI was proven to be a useful technique for differentiation between malignant and benign pulmonary nodules or masses in oncology; however, it has been never tested on MTC patients [69] (Figure 8).

(2) Liver Metastases. Liver metastases occur in $25-30 \%$ of patients with MTC $[70,71]$ and $45 \%$ of patients with advanced MTC [27]. Clinically occult liver metastases are the leading cause of failure to achieve biochemical cure with surgery [72]. On imaging, liver metastases are often small, numerous, and disseminated throughout the parenchyma; they grow slowly, and patients remain asymptomatic for a long time [63]. In one study, the size of the largest liver lesion ranged from 25 to 98 $\mathrm{mm}$ (median, $38 \mathrm{~mm}$ ) [73]. Liver metastases in MTC may have an initial miliary pattern, which makes identification difficult, and lesions may be missed [72]. Calcification of these metastases is seen in about one-third of cases [70]. Large, coarse, and irregular calcifications are more typical for MTC; however, small calcified metastases can also be seen. Extensive metastatic disease of the liver may lead to acute liver failure [74].

Ultrasound, CT, MR imaging, and PET/CT can all be used to identify hepatic metastases. The goals of imaging are to identify the location of all metastatic tumors and to determine the feasibility of local resection or the application of other therapeutic or interventional modalities. The sensitivity of US to detect and characterize metastases remains low, ranging from 50 to $76 \%$ [75], and cannot be reliably 


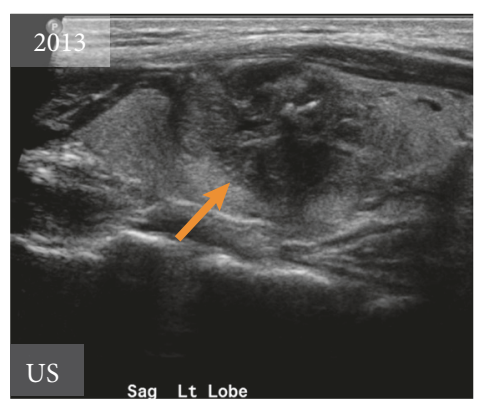

(a)

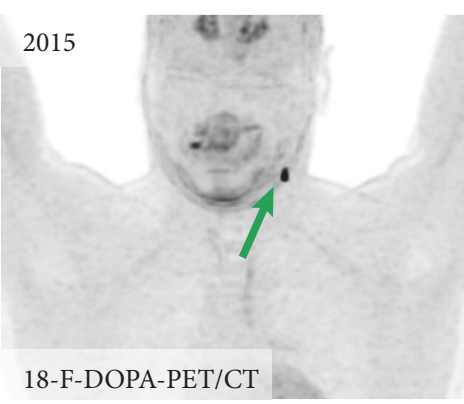

(b)

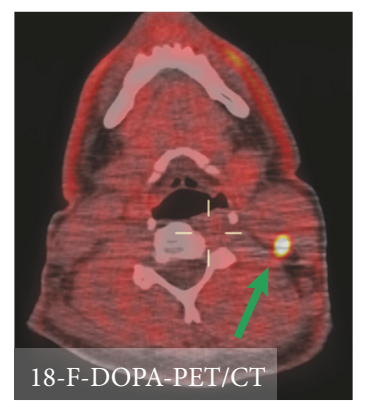

(c)

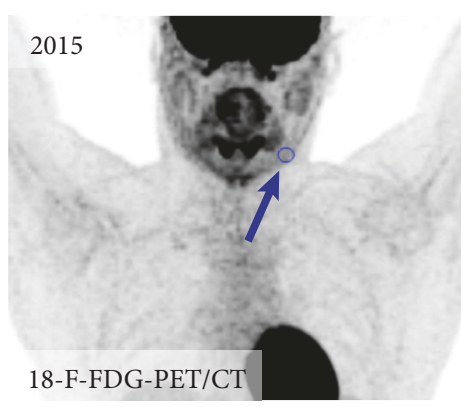

(d)

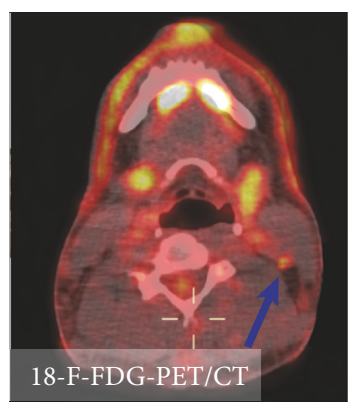

(e)

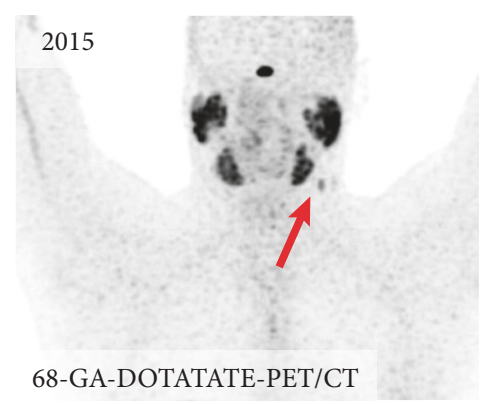

(f)

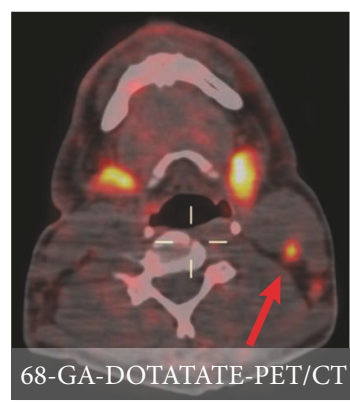

(g)

Figure 7: Mapping of the metastatic lymph nodes in medullary thyroid carcinoma. In 2015 patient underwent thyroidectomy for medullary carcinoma (a) and 2 years later presented with elevated calcitonin level (304 pg/ml). Ultrasound of the neck, CT of the neck, and MRI of the neck were all false negative (not shown); however, 18F-FDOPA-PET/CT (c), 18F-FDG-PET/CT, and 68Ga-DOTATATE-PET/CT were positive for a metastatic lymph node. Please note that patients with neck pathology should ideally be scanned with arms down (in this case the study was obtained with patient's arms up as it was a whole-body imaging for detection mediastinal metastases).

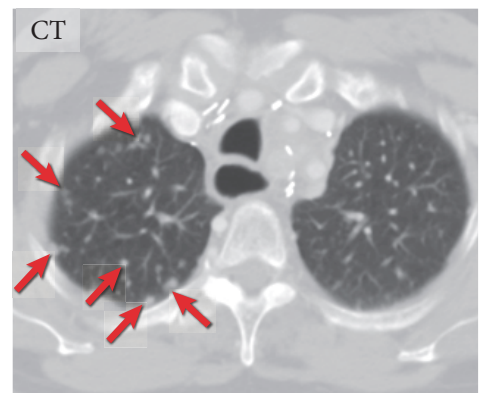

(a)

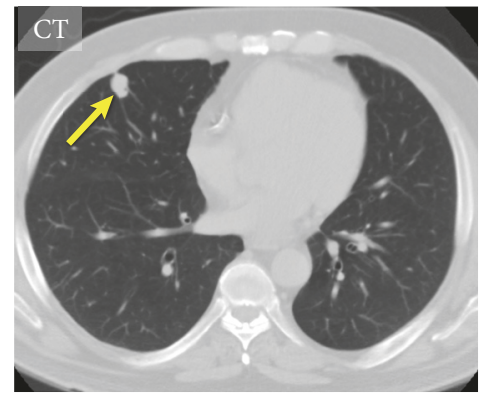

(b)

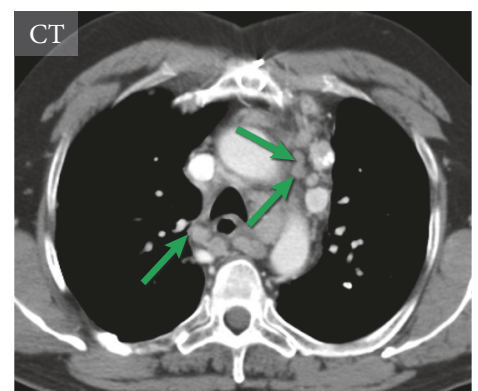

(c)

FIGURE 8: Computed tomography of the chest of different patients with metastatic medullary thyroid carcinoma. Disseminated pulmonary metastases (red arrows) (a). Solitary pulmonary metastases (yellow arrow) (b). Extensive metastatic mediastinal lymphadenopathy (green arrows) (c). 


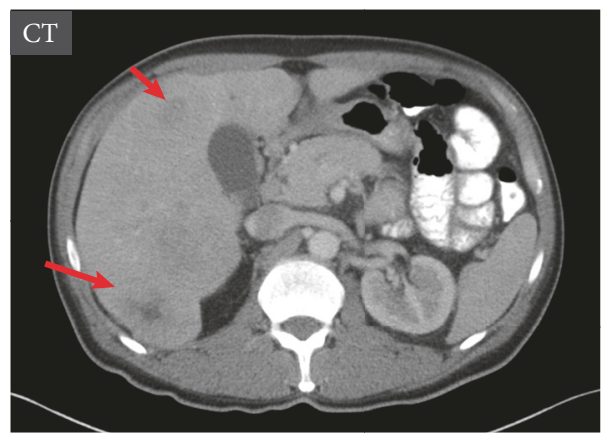

(a)

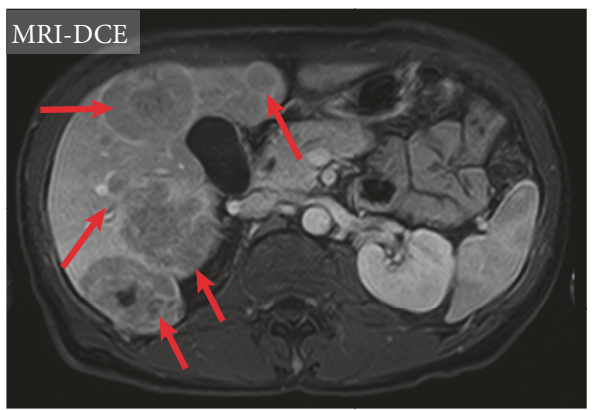

(c)

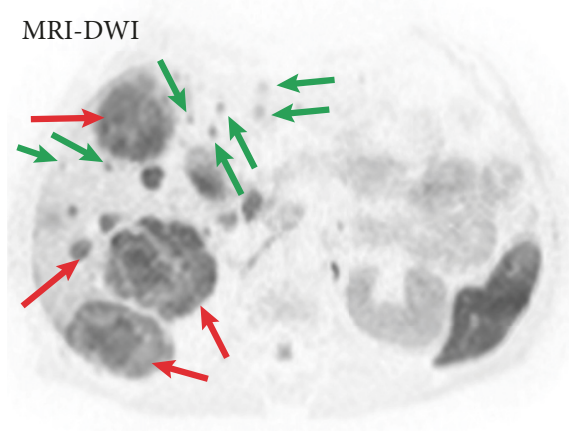

(b)

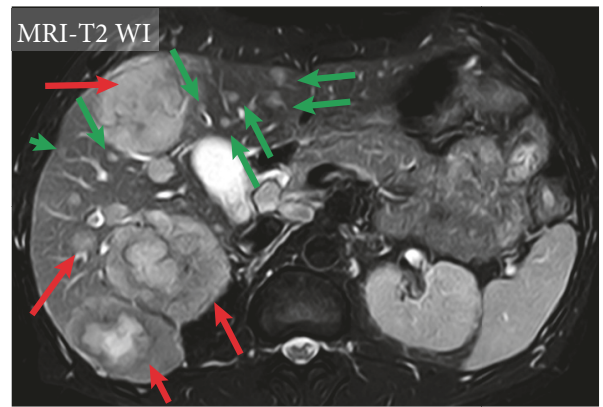

(d)

FIGURE 9: Imaging of hepatic metastases in medullary thyroid carcinoma of the same patient using contrast-enhanced computed tomography (CT) (a), magnetic resonance imaging (MRI), diffusion-weighted sequence (b); contrast-enhanced MRI, T1 weighted images (DCE)(c); MRI, T2 weighted image (d). MRI shows improved visualization of metastatic lesions compared to CT (red arrows) and can also detect very small metastases (green arrows), which may not be visible on CT.

recommended for screening or following up on patients with metastatic MTC. Four sonographic patterns of MTC hepatic metastases were described [76]; however, in era of MRI and functional imaging, it has little clinical application. The vast majority of MTC hepatic metastases were described as hyperechoic lesions and may be mistaken for hemangiomas [76, 77]. CT scans of the liver may not reveal the usual, nodular infiltration if there is diffuse intrasinusoidal spread [74]. The use of a multiphase contrast abdominal CT has proven useful in other NETs and may improve the detection of macroscopic MTC liver metastases [72]. MTC hepatic metastases are often hypervascular and have no specific radiological features on CT. Most metastases are revealed as hyperdense during hepatic arterial phase and hypodense or isodense masses on contrast-enhanced CT during the portal venous phase [78]. MRI is considered superior to CT for the detection and characterization of small liver lesions. The majority of metastases show a hypointense to isointense signal on T1WI, and an isointense to hyperintense signal on T2-WI with enhancement findings similar to those on CT. The diagnostic accuracy of standard sequences with contrast enhancement and DWI technique is equal; therefore DWI appears to be an alternative option for standard MRI protocols-it is fast and easy to perform and seems to be especially valuable for imaging in detection of liver deposits, particularly during patients' clinical follow-up period. DWI sequences improve detection rates and possibly improve the characterization of small (5-10 $\mathrm{mm}$ ) lesions in patients with NETs [79]. A single DWI scan could also be performed frequently, particularly in those patients who receive active therapy or have progressive cancer, with a potential need for changes in therapeutic management [79]. DCE MR imaging is the most sensitive imaging study for liver metastases [80]. It was found to be an effective tool in assessing and predicting the response to peptide receptor targeted radionuclide therapy in patients with NET liver metastases [81]; however, no similar studies on MTC have been performed. 18F-FDOPA is considered the best radiopharmaceutical to identify metastatic liver lesions [23]. The sensitivity of contrast-enhanced MR imaging retrospectively fused with 18F-FDG PET in the detection of liver non-MTC metastases is higher than that of 18F-FDG PET/CT [82]. Therefore, it is expected that the PET/MR imaging technique utilizing 18F-FDOPA tracer may be very helpful for detection and treatment monitoring in MTC (Figure 9).

(3) Bone Metastases. Bone metastases occur in $19-54 \%$ of patients with advanced MTC $[27,83]$. Up to now, the largest series of 188 MTC patients with bone metastases demonstrated that MTC metastases are almost uniformly multifocal, with $77 \%$ of patients having at least 6 documented lesions, with the spine and pelvis being the most common locations of the lesions. About half (48\%) of patients developed skeletalrelated events, the majority of which affected the spine (58\%), pelvis (17\%), and extremities (11\%) [83]. About 14\% of bone 


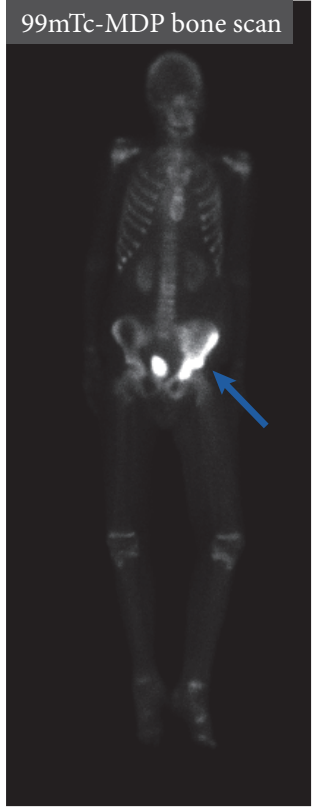

(a)

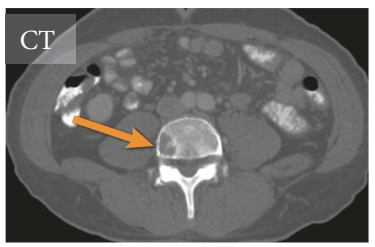

(c)

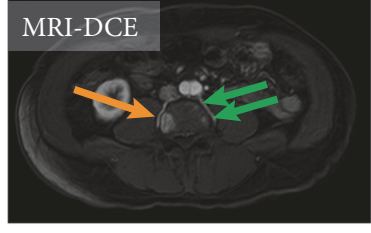

(d)

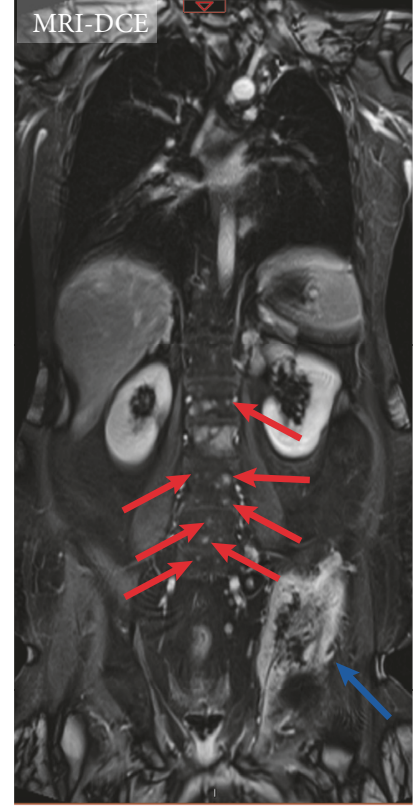

(b)

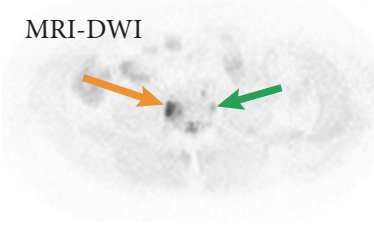

(e)

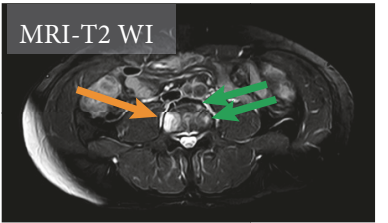

(f)

FIGURE 10: Imaging of bone metastases in medullary thyroid carcinoma on two different patients. Patient A (a-b). 99m-Tc-MDP bone scan (a) and whole-body contrast-enhanced magnetic resonance imaging (MRI), T1 weighted images (DCE) (b). MRI demonstrates improved visualization of metastatic lesions involving the left iliac bone (blue arrow) and clear visualization tiny bone metastases in the spine (red arrows). Patient B (c-f). Visualization of small bone metastases. Axial contrast-enhanced computed tomography (CT) at the level of L1 shows single lytic metastases in the right aspect of the vertebral body (orange arrow) (c). Axial contrast-enhanced MRI, T1 weighted images (DCE) (d), axial MRI diffusion-weighted sequence (e), and axial MRI, T2 weighted images (f) can detect additional small spinal metastases, which are not visualized on CT (green arrows).

metastases were diagnosed before evidence of nonosseous distant metastases, $24 \%$ had concomitant bone metastases identified at the time of distant metastases diagnosis, and $62 \%$ had bone metastases diagnosed after nonosseous distant metastases diagnosis [83]. The radiological appearance of MTC bone metastases is not specific. On CT, lesions may be osteolytic, osteoblastic, or mixed [80]. On MR imaging, they usually have low signal T1-WI and enhance after contrast administration, similar to other metastases. Bone scan of osteoblastic metastases usually demonstrates focal increased uptake of radioactivity, whereas lytic bone metastases may appear as photopenic areas unless they cause a pathologic fracture, which manifests itself with increased tracer uptake.

Up to now, there is no clear answer to the question as to which modality is the most accurate for the identification of bone MTC metastases, as no recent studies evaluating the sensitivity and specificity of different imaging modalities in MTC bone metastases and the assessment of the response to the therapy have been performed. Two early studies showed contradictory results: Mirallié et al. (2005) demonstrated a higher sensitivity of MR imaging for detecting bone metastases compared with bone scintigraphy, while Giraudet et al. (2007) claimed a better sensitivity of bone scans [27, 84]. In another small study, 68Ga-DOTATATE PET/CT demonstrated a high sensitivity in detecting bone lesions and potentially it may be considered a substitute for a bone scan and MRI [33]. The European Organization for Research and Treatment of Cancer (EORTC) imaging group stresses the superiority of MR imaging over bone scintigraphy for the detection of all bone metastases and suggests the use of whole-body MRI and 18F-FDOPA PET/CT for the detection of MTC bone lesions [85] (Figure 10).

3.3. MTC in Patients with MEN2 Syndromes. Patients with MEN2 should be evaluated for pheochromocytoma before thyroid surgery for MTC. Therefore, serum calcium, phosphate, and parathyroid hormone levels, as well as 


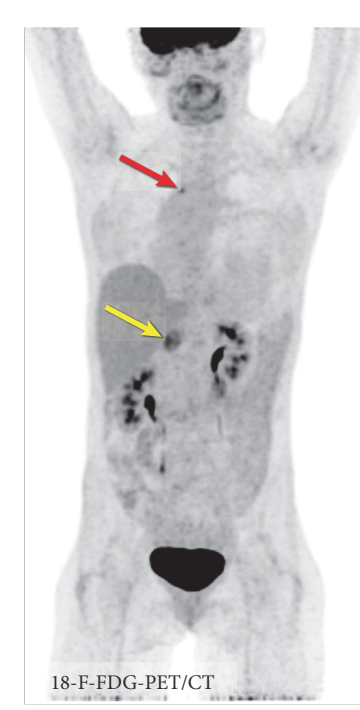

(a)

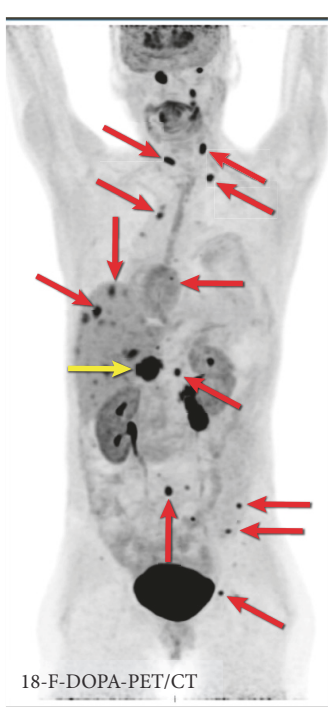

(b)

FIGURE 11: Imaging in MEN syndrome. 18F-FDG PET/CT (a) and 18F-FDOPA PET/CT (b) in MEN2B syndrome with metastatic pheochromocytoma (fractionated norepinephrine: $994 \mathrm{pg} / \mathrm{ml}$, normal: 18-112 pg/ml; fractionated metanephrine: 1099 pg/ml, normal: 12 $61 \mathrm{pg} / \mathrm{ml}$ ) and metastatic medullary carcinoma (calcitonin: 5575, normal: $<8 \mathrm{pg} / \mathrm{ml}$ ). In this case, the primary pheochromocytoma is seen in all images (yellow arrow); however, metastatic lesions are best visualized on 18F-FDOPA PET/CT (red arrows). Imaging cannot differentiate between medullary carcinoma metastases and metastases originating from pheochromocytoma.

catecholamine metabolites in plasma and urine, are part of the routine laboratory workup. Imaging cannot differentiate between medullary carcinoma metastases and metastases originating from pheochromocytoma (Figure 11).

\section{Tumor Response Criteria in MTC}

Accurate staging and evaluation of treatment response are critical for optimal treatment decisions in MTC. Response Evaluation Criteria in Solid Tumors (RECIST), the most commonly used universal system for the quantification of tumor response in oncologic imaging, has been used to assess the effects of MTC treatment medications in different studies and clinical trials [86-89]. However, it appears that the RECIST system is not ideal for MTC. Lesions smaller than $1 \mathrm{~cm}$ are considered "nonmeasurable," and miliary MTC metastases cannot be assessed. Blastic bone metastases also remain "nonmeasurable" and, given the high rate of bone metastases in MTC, these lesions cannot be evaluated [85]. The majority of the studies devoted to MTC showed no correlation between RECIST response and biochemical markers-patients who responded to the therapy predominantly based on CNT level showed stable disease based on RECIST criteria despite apparent biochemical responses $[13,87,88,90-92]$. Biological markers (CNT and CEA levels and doubling times) alone cannot be used as tumor response criteria, and they do not address the heterogeneity of response at different sites as therapy-resistant clones develop. A dedicated system for assessment of the MTC response is needed, which probably should include conventional and functional imaging including 18F-FDOPA and 18F-FDG PET/CT with relation to the biochemical markers.

\section{Conclusions}

Functional imaging, primarily PET/CT with 18F-FDOPA and $18 \mathrm{~F}-\mathrm{FDG}$, plays a crucial role in the evaluation and management of MTC and has proven to be an efficient tool for the detection of metastases in patients with elevated CTN levels. Future research is needed to evaluate the role of advanced imaging techniques such as DWI, DCE, and hybrid PET/MR for MTC and to clarify the CTN cutoff for the follow-up taking into account the recent progress in imaging techniques. It can be suggested that any patient's elevated postoperative CTN should be considered as metastatic disease of unknown location and should prompt comprehensive radiological evaluation. These patients may benefit from 18F-FDOPA PET/CT with following targeted imaging depending on specific organ involvement.

Modern imaging techniques can provide an accurate assessment of a response to therapies and facilitate the translation of basic science into clinical application in clinical trials. Although a number of new imaging modalities evolved over the last decades and are effectively used in different oncological applications, the clinical and research use of these novel techniques are still limited in the comprehensive evaluation of MTC, and new studies focusing on MRI techniques are needed. It is important to develop a dedicated system for the assessment of the MTC response, which probably should include conventional and molecular imaging including $18 \mathrm{~F}$ FDOPA and 18F-FDG PET with relation to the biochemical 
markers, as it appears that the RECIST system is not ideal for MTC.

\section{Conflicts of Interest}

The authors declare that they have no conflicts of interest.

\section{Acknowledgments}

This study was funded by the National Institutes of Health (grant number Z1AHD008735, awarded to Karel Pacak). We would like to express our gratitude to Irina Nefedova, a Ukrainian artist, for her help in preparing the illustrations.

\section{References}

[1] M. Nilsson and D. Williams, "On the origin of cells and derivation of thyroid cancer: C cell story revisited," European Thyroid Journal, vol. 5, no. 2, pp. 79-93, 2016.

[2] M. Roy, H. Chen, and R. S. Sippel, "Current understanding and management of medullary thyroid cancer," The Oncologist, vol. 18, no. 10, pp. 1093-1100, 2013.

[3] S. Roman, R. Lin, and J. A. Sosa, "Prognosis of medullary thyroid carcinoma: demographic, clinical, and pathologic predictors of survival in 1252 cases," Cancer, vol. 107, no. 9, pp. 21342142, 2006.

[4] G. Treglia, V. Rufini, M. Salvatori, A. Giordano, and L. Giovanella, "PET imaging in recurrent medullary thyroid carcinoma," International Journal of Molecular Imaging, vol. 2012, Article ID 324686, 9 pages, 2012.

[5] P. Trimboli, L. Guidobaldi, M. Bongiovanni, A. Crescenzi, M. Alevizaki, and L. Giovanella, "Use of fine-needle aspirate calcitonin to detect medullary thyroid carcinoma: a systematic review," Diagnostic Cytopathology, vol. 44, no. 1, pp. 45-51, 2016.

[6] J. P. B. O'Connor, C. J. Rose, A. Jackson et al., "DCE-MRI biomarkers of tumour heterogeneity predict CRC liver metastasis shrinkage following bevacizumab and FOLFOX-6," British Journal of Cancer, vol. 105, no. 1, pp. 139-145, 2011.

[7] H. W. Goo, "Whole-body MRI in children: Current imaging techniques and clinical applications," Korean Journal of Radiology, vol. 16, no. 5, pp. 973-985, 2015.

[8] E. Van Cutsem, H. Verheul, P. Flamen et al., "Imaging in colorectal cancer: progress and challenges for the clinicians," Cancers, vol. 8, no. 9, 2016.

[9] B. Taouli, A. J. Beer, T. Chenevert et al., "Diffusion-weighted imaging outside the brain: Consensus statement from an ISMRM-sponsored workshop," Journal of Magnetic Resonance Imaging, vol. 44, no. 3, pp. 521-540, 2016.

[10] A. R. Padhani, D.-M. Koh, and D. J. Collins, "Whole-body diffusion-weighted MR imaging in cancer: current status and research directions," Radiology, vol. 261, no. 3, pp. 700-718, 2011.

[11] Y. S. Sung, B. Park, Y. Choi et al., "Dynamic contrast-enhanced MRI for oncology drug development," Journal of Magnetic Resonance Imaging, vol. 44, no. 2, pp. 251-264, 2016.

[12] S. I. Sherman, “Targeted therapies for thyroid tumors," Modern Pathology, vol. 24, Suppl 2, pp. S44-S52, 2011.

[13] E. T. Lam, M. D. Ringel, R. T. Kloos et al., "Phase II clinical trial of sorafenib in metastatic medullary thyroid cancer," Journal of Clinical Oncology, vol. 28, no. 14, pp. 2323-2330, 2010.
[14] C. Riola-Parada, L. García-Cañamaque, V. Pérez-Dueñas, M. Garcerant-Tafur, and J. Carreras-Delgado, "Simultaneous $\mathrm{PET} / \mathrm{MRI}$ vs PET/CT in oncology. A systematic review," Revista Española de Medicina Nuclear e Imagen Molecular, vol. 35, no. 5, pp. 306-312, 2016.

[15] S. A. Wells Jr., S. L. Asa, H. Dralle et al., "Revised American Thyroid Association guidelines for the management of medullary thyroid carcinoma," Thyroid, vol. 25, no. 6, pp. 567-610, 2015.

[16] N. D. Riddle and M. M. Bui, "Fibrous dysplasia," Archives of Pathology \& Laboratory Medicine, vol. 137, no. 1, pp. 134-138, 2013.

[17] G. Treglia, M. F. Villani, A. Giordano, and V. Rufini, "Detection rate of recurrent medullary thyroid carcinoma using fluorine18 fluorodeoxyglucose positron emission tomography: A metaanalysis," Endocrine Journal, vol. 42, no. 3, pp. 535-545, 2012.

[18] G. Treglia, F. Cocciolillo, F. Di Nardo et al., "Detection rate of recurrent medullary thyroid carcinoma using fluorine-18 dihydroxyphenylalanine positron emission tomography: a metaanalysis," Academic Radiology, vol. 19, no. 10, pp. 1290-1299, 2012.

[19] A. Sesti, M. Mayerhoefer, M. Weber et al., "Relevance of calcitonin cut-off in the follow-up of medullary thyroid carcinoma for conventional imaging and 18-fluorine-fluorodihydroxyphenylalanine PET,' Anticancer Reseach, vol. 34, no. 11, pp. 6647-6654, 2014.

[20] A. R. Romero-Lluch, J. I. Cuenca-Cuenca, R. Guerrero-Vázquez et al., "Diagnostic utility of PET/CT with 18F-DOPA and 18FFDG in persistent or recurrent medullary thyroid carcinoma: the importance of calcitonin and carcinoembryonic antigen cutoff," European Journal of Nuclear Medicine and Molecular Imaging, 2017.

[21] M. Luster, W. Karges, K. Zeich et al., "Clinical value of 18fluorine-fluorodihydroxyphenylalanine positron emission tomography/computed tomography in the follow-up of medullary thyroid carcinoma," Thyroid, vol. 20, no. 5, pp. 527-533, 2010.

[22] H. H. G. Verbeek, J. T. M. Plukker, K. P. Koopmans et al., "Clinical relevance of 18F-FDG PET and 18F-DOPA PET in recurrent medullary thyroid carcinoma," Journal of Nuclear Medicine, vol. 53, no. 12, pp. 1863-1871, 2012.

[23] G. Treglia, P. Castaldi, M. F. Villani et al., "Comparison of 18FDOPA, 18F-FDG and 68Ga-somatostatin analogue PET/CT in patients with recurrent medullary thyroid carcinoma," European Journal of Nuclear Medicine and Molecular Imaging, vol. 39, no. 4, pp. 569-580, 2012.

[24] M. Marzola, M. Pelizzo, M. Ferdeghini et al., "Dual PET/CT with (18)F-DOPA and (18)F-FDG in metastatic medullary thyroid carcinoma and rapidly increasing calcitonin levels: Comparison with conventional imaging," European Journal of Surgical Oncology, vol. 36, no. 4, pp. 414-421, 2010.

[25] H. W. Jang, J. Y. Choi, J. I. Lee et al., "Localization of medullary thyroid carcinoma after surgery using (11)Cmethionine PET/CT: comparison with (18)F-FDG PET/CT," Endocrine Journal, vol. 57, no. 12, pp. 1045-1054, 2010.

[26] S. C. Ong, H. Schöder, S. G. Patel et al., "Diagnostic accuracy of 18F-FDG PET in restaging patients with medullary thyroid carcinoma and elevated calcitonin levels," Journal of Nuclear Medicine, vol. 48, no. 4, pp. 501-507, 2007.

[27] A. L. Giraudet, D. Vanel, S. Leboulleux, A. Aupérin, C. Dromain, and L. Chami, "Imaging medullary thyroid carcinoma with persistent elevated calcitonin levels," The Journal of Clinical 
Endocrinology and Metabolism, vol. 92, no. 11, pp. 4185-4190, 2007.

[28] A. Oudoux, P. Salaun, C. Bournaud et al., "Sensitivity and prognostic value of positron emission tomography with F18-fluorodeoxyglucose and sensitivity of immunoscintigraphy in patients with medullary thyroid carcinoma treated with anticarcinoembryonic antigen-targeted radioimmunotherapy," The Journal of Clinical Endocrinology \& Metabolism, vol. 92, no. 12, pp. 4590-4597, 2007.

[29] G. Treglia, C. Aktolun, A. Chiti et al., "The 2015 Revised American Thyroid Association guidelines for the management of medullary thyroid carcinoma: the "evidence-based" refusal to endorse them by EANM due to the "not evidence-based" marginalization of the role of Nuclear Medicine," European Journal of Nuclear Medicine and Molecular Imaging, vol. 43, no. 8, pp. 1486-1490, 2016.

[30] E. Skoura, "Depicting medullary thyroid cancer recurrence: The past and the future of nuclear medicine imaging," International Journal of Endocrinology and Metabolism, vol. 11, no. 4, Article ID e8156, 2013.

[31] L. Y. I. Yamaga, M. L. Cunha, G. C. Campos Neto et al., “(68)GaDOTATATE PET/CT in recurrent medullary thyroid carcinoma: a lesion-by-lesion comparison with (111)In-octreotide SPECT/CT and conventional imaging," European Journal of Nuclear Medicine and Molecular Imaging, vol. 44, no. 10, pp. 1695-1701, 2017.

[32] G. Treglia, A. Tamburello, and L. Giovanella, "Detection rate of somatostatin receptor pet in patients with recurrent medullary thyroid carcinoma: A systematic review and a meta-analysis," Hormones, vol. 16, no. 4, pp. 362-372, 2017.

[33] L. A. Castroneves, G. Coura Filho, R. M. de Freitas et al., "Comparison of $68 \mathrm{Ga}$ PET/CT to other imaging studies in medullary thyroid cancer: superiority in detecting bone metastases," The Journal of Clinical Endocrinology \& Metabolism, vol. 103, no. 9, pp. 3250-3259, 2018.

[34] J. C. Reubi, "Targeting CCK receptors in human cancers," Current Topics in Medicinal Chemistry, vol. 7, no. 12, pp. 12391242, 2007.

[35] M. Bläker, A. de Weerth, M. Tometten et al., "Expression of the cholecystokinin 2-receptor in normal human thyroid gland and medullary thyroid carcinoma," European Journal of Endocrinology, vol. 146, no. 1, pp. 89-96, 2002.

[36] E. Von Guggenberg, C. Rangger, J. Sosabowski et al., "Preclinical evaluation of radiolabeled DOTA-derivatized cyclic minigastrin analogs for targeting cholecystokinin receptor expressing malignancies," Molecular Imaging and Biology, vol. 14, no. 3, pp. 366-375, 2012.

[37] M. Gotthardt, M. P. Béhé, D. Beuter et al., "Improved tumour detection by gastrin receptor scintigraphy in patients with metastasised medullary thyroid carcinoma," European Journal of Nuclear Medicine and Molecular Imaging, vol. 33, no. 11, pp. 1273-1279, 2006.

[38] T. M. Behr and M. P. Béhé, "Cholecystokinin-B/gastrin receptor-targeting peptides for staging and therapy of medullary thyroid cancer and other cholecystokinin-B receptor-expressing malignancies," Seminars in Nuclear Medicine, vol. 32, no. 2, pp. 97-109, 2002.

[39] I. Ilias, C. Divgi, and K. Pacak, "Current role of metaiodobenzylguanidine in the diagnosis of pheochromocytoma and medullary thyroid cancer," Seminars in Nuclear Medicine, vol. 41, no. 5, pp. 364-368, 2011.
[40] Z. Gao, H. J. Biersack, S. Ezziddin, T. Logvinski, and R. An, "The role of combined imaging in metastatic medullary thyroid carcinoma: 111In-DTPA-octreotide and 131I/123I-MIBG as predictors for radionuclide therapy," Journal of Cancer Research and Clinical Oncology, vol. 130, no. 11, pp. 649-656, 2004.

[41] P. L. Jager, W. Vaalburg, J. Pruim et al., "Radiolabeled amino acids: basic aspects and clinical applications in oncology," The Journal of Nuclear Medicine, vol. 42, no. 3, pp. 432-445, 2001.

[42] C. Bodet-Milin, A. Faivre-Chauvet, T. Carlier et al., "ImmunoPET using anticarcinoembryonic antigen bispecific antibody and 68Ga-labeled peptide in metastatic medullary thyroid carcinoma: Clinical optimization of the pretargeting parameters in a first-in-human trial," Journal of Nuclear Medicine, vol. 57, no. 10, pp. 1505-1511, 2016.

[43] E. Kebebew, P. H. G. Ituarte, A. E. Siperstein, Q.-Y. Duh, and O. H. Clark, "Medullary thyroid carcinoma: clinical characteristics, treatment, prognostic factors, and a comparison of staging systems," Cancer, vol. 88, no. 5, pp. 1139-1148, 2000.

[44] S. Lee, J. H. Shin, B.-K. Han, and E. Y. Ko, "Medullary thyroid carcinoma: Comparison with papillary thyroid carcinoma and application of current sonographic criteria," American Journal of Roentgenology, vol. 194, no. 4, pp. 1090-1094, 2010.

[45] M. Fukushima, Y. Ito, M. Hirokawa et al., "Excellent prognosis of patients with nonhereditary medullary thyroid carcinoma with ultrasonographic findings of follicular tumor or benign nodule," World Journal of Surgery, vol. 33, no. 5, pp. 963-968, 2009.

[46] P. Trimboli, L. Giovanella, S. Valabrega et al., "Ultrasound features of medullary thyroid carcinoma correlate with cancer aggressiveness: a retrospective multicenter study," Journal of Experimental \& Clinical Cancer Research, vol. 33, p. 87, 2014.

[47] B. R. Haugen, E. K. Alexander, K. C. Bible et al., "2015 american thyroid association management guidelines for adult patients with thyroid nodules and differentiated thyroid cancer: the american thyroid association guidelines task force on thyroid nodules and differentiated thyroid cancer," Thyroid, vol. 26, no. 1, pp. 1-133, 2016.

[48] P. Valderrabano, D. L. Klippenstein, J. B. Tourtelot et al., "New american thyroid association sonographic patterns for thyroid nodules perform well in medullary thyroid carcinoma: institutional experience, systematic review, and meta-analysis," Thyroid, vol. 26, no. 8, pp. 1093-1100, 2016.

[49] G. Essig, K. Porter, D. Schneider et al., "Fine needle aspiration and medullary thyroid carcinoma: the risk of inadequate preoperative evaluation and initial surgery when relying upon FNAB cytology alone," Endocrine Practice, vol. 19, no. 6, pp. 920-927, 2013.

[50] M. J. M. Bugalho, J. R. Santos, and L. Sobrinho, "Preoperative diagnosis of medullary thyroid carcinoma: Fine needle aspiration cytology as compared with serum calcitonin measurement," Journal of Surgical Oncology, vol. 91, no. 1, pp. 56-60, 2005.

[51] R. T. Kloos, R. J. Monroe, S. T. Traweek, R. B. Lanman, and G. C. Kennedy, "A genomic alternative to identify medullary thyroid cancer preoperatively in thyroid nodules with indeterminate cytology," Thyroid, vol. 26, no. 6, pp. 785-793, 2016.

[52] G. Costante, C. Durante, Z. Francis, M. Schlumberger, and S. Filetti, "Determination of calcitonin levels in C-cell disease: clinical interest and potential pitfalls," Nature Clinical Practice Endocrinology \& Metabolism, vol. 5, no. 1, pp. 35-44, 2009.

[53] E. G. Grant, F. N. Tessler, J. K. Hoang et al., “Thyroid ultrasound reporting lexicon: White paper of the ACR thyroid imaging, 
reporting and data system (TIRADS) committee," Journal of the American College of Radiology, vol. 12, no. 12, pp. 1272-1279, 2015.

[54] D. Taïeb, E. Kebebew, F. Castinetti, C. C. Chen, J.-F. Henry, and K. Pacak, "Diagnosis and preoperative imaging of multiple endocrine neoplasia type 2: Current status and future directions," Clinical Endocrinology, vol. 81, no. 3, pp. 317-328, 2014.

[55] M. A. Kouvaraki, S. E. Shapiro, B. D. Fornage et al., "Role of preoperative ultrasonography in the surgical management of patients with thyroid cancer," Surgery, vol. 134, no. 6, pp. 946954, 2003.

[56] A. Machens, U. Schneyer, H. Holzhausen, and H. Dralle, "Prospects of remission in medullary thyroid carcinoma according to basal calcitonin level," The Journal of Clinical Endocrinology \& Metabolism, vol. 90, no. 4, pp. 2029-2034, 2005.

[57] A. Machens and H. Dralle, "Biomarker-based risk stratification for previously untreated medullary thyroid cancer," The Journal of Clinical Endocrinology \& Metabolism, vol. 95, no. 6, pp. 26552663, 2010.

[58] G. Pellegriti, S. Leboulleux, E. Baudin et al., "Long-term outcome of medullary thyroid carcinoma in patients with normal postoperative medical imaging," British Journal of Cancer, vol. 88, no. 10, pp. 1537-1542, 2003.

[59] M. R. Pelizzo, F. Torresan, A. Da Roit et al., "Mild to moderate increase of serum calcitonin levels only in presence of large medullary thyroid cancer deposits," Revista Española de Medicina Nuclear e Imagen Molecular, vol. 34, no. 6, pp. 378-382, 2015.

[60] T. J. Fraum, K. J. Fowler, and J. McConathy, "PET/MRI: emerging clinical applications in oncology," Academic Radiology, vol. 23, no. 2, pp. 220-236, 2016.

[61] B. Nelkin, "Recent advances in the biology and therapy of medullary thyroid carcinoma," F1000Research, vol. 6, p. 2184, 2017.

[62] J. K. Hoang, K. L. Wai, M. Lee, D. Johnson, and S. Farrell, "US features of thyroid malignancy: pearls and pitfalls," RadioGraphics, vol. 27, no. 3, pp. 847-860, 2007.

[63] F. Raue and K. Frank-Raue, "Long-term follow-up in medullary thyroid carcinoma," Recent Results in Cancer Research, vol. 204, pp. 207-225, 2015.

[64] J. M. Jimenez, S. O. Casey, M. Citron, and A. Khan, "Calcified pulmonary metastases from medullary carcinoma of the thyroid," Computerized Medical Imaging and Graphics, vol. 19, no. 4, pp. 325-328, 1995.

[65] N. Ventura, E. Marchiori, G. Zanetti et al., "Medullary thyroid carcinoma with micronodular lung metastases: a case report with an emphasis on the imaging findings," Case Reports in Medicine, vol. 2010, Article ID 616580, 5 pages, 2010.

[66] G. Mazoyer, J. F. Cordier, J. M. Zabern et al., "Pulmonary metastases in medullary cancers of the thyroid. Study of 4 cases. Originality of the lymphangitic form with amyloid stroma," Revue des Maladies Respiratoires, vol. 3, no. 3, pp. 139-143, 1986.

[67] B. G. Brogdon, C. A. Kelsey, and R. D. Moseley Jr., "Factors affecting perception of pulmonary lesions," Radiologic Clinics of North America, vol. 21, no. 4, pp. 633-654, 1983.

[68] Y. Kurihara, S. Matsuoka, T. Yamashiro et al., "MRI of pulmonary nodules," American Journal of Roentgenology, vol. 202, no. 3, pp. W210-W216, 2014.

[69] L. Chen, J. Zhang, J. Bao et al., "Meta-analysis of diffusionweighted MRI in the differential diagnosis of lung lesions," Journal of Magnetic Resonance Imaging, vol. 37, no. 6, pp. 13511358, 2013.
[70] C. H. McDonnell, E. K. Fishman, and E. A. Zerhouni, "CT demonstration of calcified liver metastases in medullary thyroid carcinoma," Journal of Computer Assisted Tomography, vol. 10, no. 6, pp. 976-978, 1986.

[71] M. S. Bankoff, G. A. Turkman, and D. Scarborough, "CT appearance of liver metastases from medullary carcinoma of the thyroid," Journal of Computer Assisted Tomography, vol. 11, no. 6, pp. 1102-1103, 1987.

[72] D. W. Ball, "Medullary thyroid cancer: monitoring and therapy," Endocrinology and Metabolism Clinics of North America, vol. 36, no. 3, pp. 823-837, 2007.

[73] J. Fromigue, T. De Baere, E. Baudin et al., "Chemoembolization for liver metastases from medullary thyroid carcinoma," The Journal of Clinical Endocrinology \& Metabolism, vol. 91, no. 7, pp. 2496-2499, 2006.

[74] E. C. Gorospe and J. Badamas, "Acute liver failure secondary to metastatic medullary thyroid cancer," Case Reports in Hepatology, vol. 2011, Article ID 603757, 4 pages, 2011.

[75] V. Cantisani, H. Grazhdani, C. Fioravanti et al., "Liver metastases: Contrast-enhanced ultrasound compared with computed tomography and magnetic resonance," World Journal of Gastroenterology, vol. 20, no. 29, pp. 9998-10007, 2014.

[76] J. Leclere, S. Sidibé, N. Lassau et al., "Ultrasonographic aspects of hepatic metastases of thyroid medullary cancers," European Journal of Radiology, vol. 77, no. 2, pp. 99-103, 1996.

[77] S. Delorme and F. Raue, "Medullary thyroid carcinoma: Imaging," Recent Results in Cancer Research, vol. 204, pp. 91-116, 2015.

[78] R. M. Gore, K. H. Thakrar, D. R. Wenzke, G. M. Newmark, U. K. Mehta, and J. W. Berlin, "That liver lesion on MDCT in the oncology patient: Is it important?” Cancer Imaging, vol. 12, no. 2, pp. 373-384, 2012.

[79] A. J. Sankowski, J. B. Ćwikła, M. L. Nowicki et al., "The clinical value of MRI using single-shot echoplanar DWI to identify liver involvement in patients with advanced gastroenteropancreaticneuroendocrine tumors (GEP-NETs), compared to FSE T2 and FFE T1 weighted image after i.v. Gd-EOB-DTPA contrast enhancement," Medical Science Monitor, vol. 18, no. 5, pp. MT33-MT40, 2012.

[80] D. Ganeshan, E. Paulson, C. Duran, M. E. Cabanillas, N. L. Busaidy, and C. Charnsangavej, "Current update on medullary thyroid carcinoma," American Journal of Roentgenology, vol. 201, no. 6, pp. W867-W876, 2013.

[81] K. Miyazaki, M. R. Orton, R. L. Davidson et al., "Neuroendocrine tumor liver metastases: Use of dynamic contrastenhanced MR imaging to monitor and predict radiolabeled octreotide therapy response," Radiology, vol. 263, no. 1, pp. 139148, 2012.

[82] O. F. Donati, T. F. Hany, C. S. Reiner et al., "Value of retrospective fusion of PET and MR images in detection of hepatic metastases: comparison with 18F-FDG PET/CT and Gd-EOB-DTPAenhanced MRI," Journal of Nuclear Medicine, vol. 51, no. 5, pp. 692-699, 2010.

[83] J. Y. Xu, W. A. Murphy Jr., D. R. Milton et al., "Bone metastases and skeletal-related events in medullary thyroid carcinoma," The Journal of Clinical Endocrinology and Metabolism, Article ID jc20162815, 2016.

[84] E. Mirallié, J. P. Vuillez, S. Bardet et al., "High frequency of bone/bone marrow involvement in advanced medullary thyroid cancer," The Journal of Clinical Endocrinology \& Metabolism, vol. 90, no. 2, pp. 779-788, 2005.

[85] F. E. Lecouvet, J. N. Talbot, C. Messiou, P. Bourguet, Y. Liu, and N. M. de Souza, "Monitoring the response of bone metastases 
to treatment with Magnetic Resonance Imaging and nuclear medicine techniques: a review and position statement by the European Organisation for Research and Treatment of Cancer imaging group," European Journal of Cancer, vol. 50, no. 15, pp. 2519-2531, 2014.

[86] E. A. Eisenhauer, P. Therasse, J. Bogaerts et al., "New response evaluation criteria in solid tumours: revised RECIST guideline (version 1.1)," European Journal of Cancer, vol. 45, no. 2, pp. 228247, 2009.

[87] M. Schlumberger, B. Jarzab, M. E. Cabanillas et al., "A Phase II Trial of the Multitargeted Tyrosine Kinase Inhibitor Lenvatinib (E7080) in Advanced Medullary Thyroid Cancer," Clinical Cancer Research, vol. 22, no. 1, pp. 44-53, 2016.

[88] L. A. De Castroneves, M. V. Negrão, R. M. C. De Freitas et al., "Sorafenib for the treatment of progressive metastatic medullary thyroid cancer: Efficacy and safety analysis," Thyroid, vol. 26, no. 3, pp. 414-419, 2016.

[89] J. Fromigué, T. De Baere, E. Baudin, C. Dromain, S. Leboulleux, and M. Schlumberger, "Chemoembolization for liver metastases from medullary thyroid carcinoma," The Journal of Clinical Endocrinology \& Metabolism, vol. 91, no. 7, pp. 2496-2499, 2006.

[90] E. Grande Pulido, B. Castelo, P. J. Fonseca et al., "Efficacy and tolerability of sunitinib in patients with advanced thyroid cancer out of a trial: A Spanish multicenter cohort," Journal of Clinical Oncology, vol. 29, no. 15_suppl, p. e16024, 2011.

[91] C. N. Chougnet, I. Borget, S. Leboulleux et al., "Vandetanib for the treatment of advanced medullary thyroid cancer outside a clinical trial: Results from a french cohort," Thyroid, vol. 25, no. 4, pp. 386-391, 2015.

[92] S. A. Wells Jr., B. G. Robinson, R. F. Gagel et al., "Vandetanib in patients with locally advanced or metastatic medullary thyroid cancer: a randomized, double-blind phase III trial," Journal of Clinical Oncology, vol. 30, no. 2, pp. 134-141, 2012. 


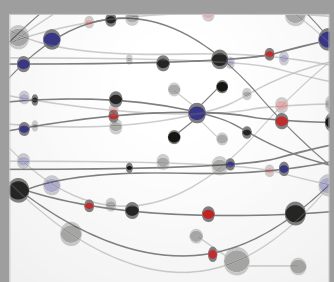

The Scientific World Journal
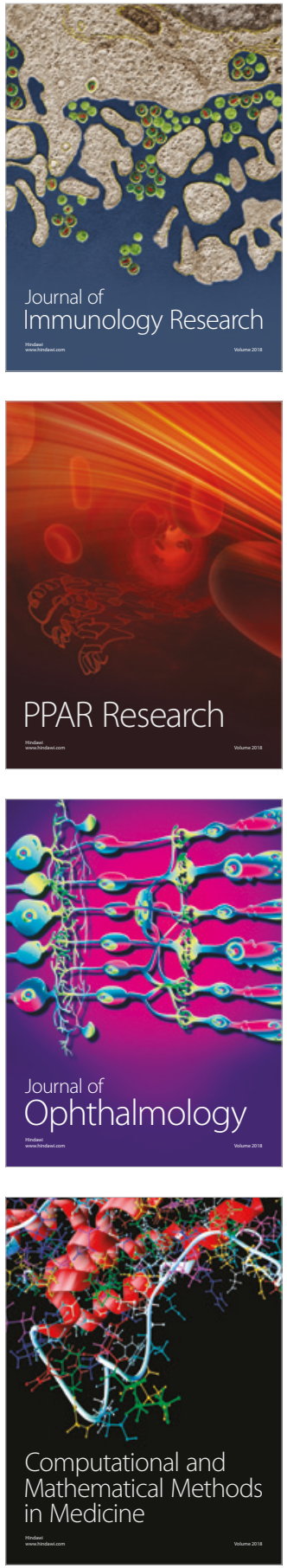

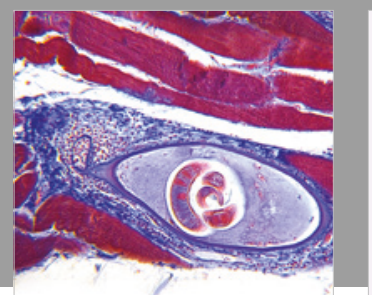

Gastroenterology Research and Practice

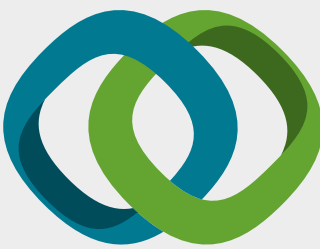

\section{Hindawi}

Submit your manuscripts at

www.hindawi.com
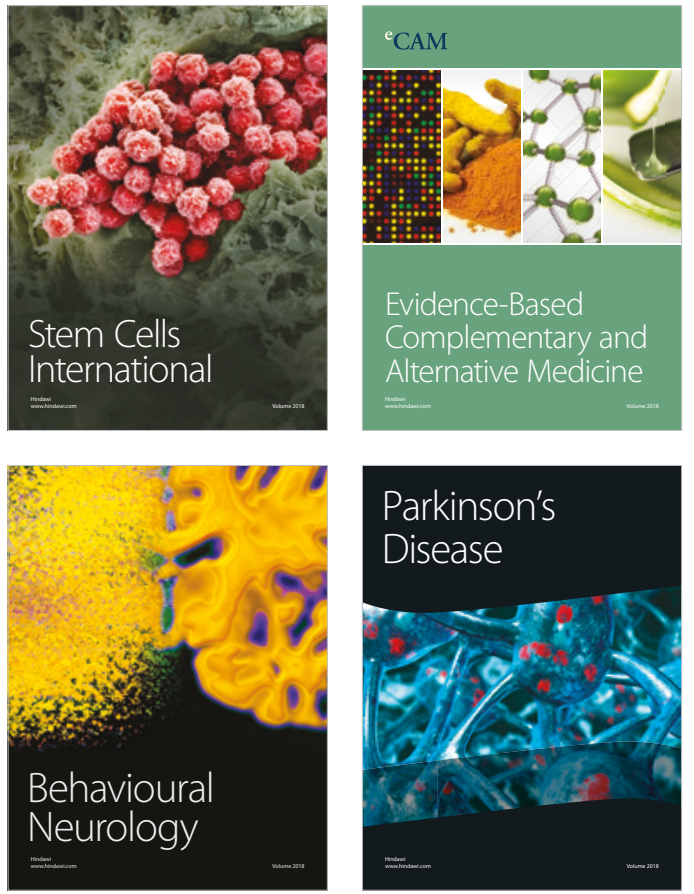

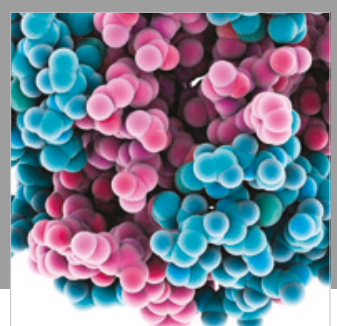

ournal of

Diabetes Research

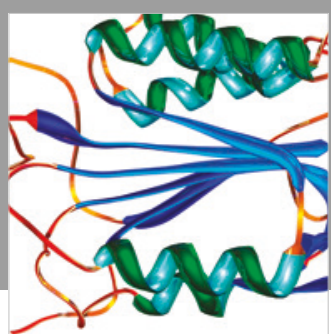

Disease Markers
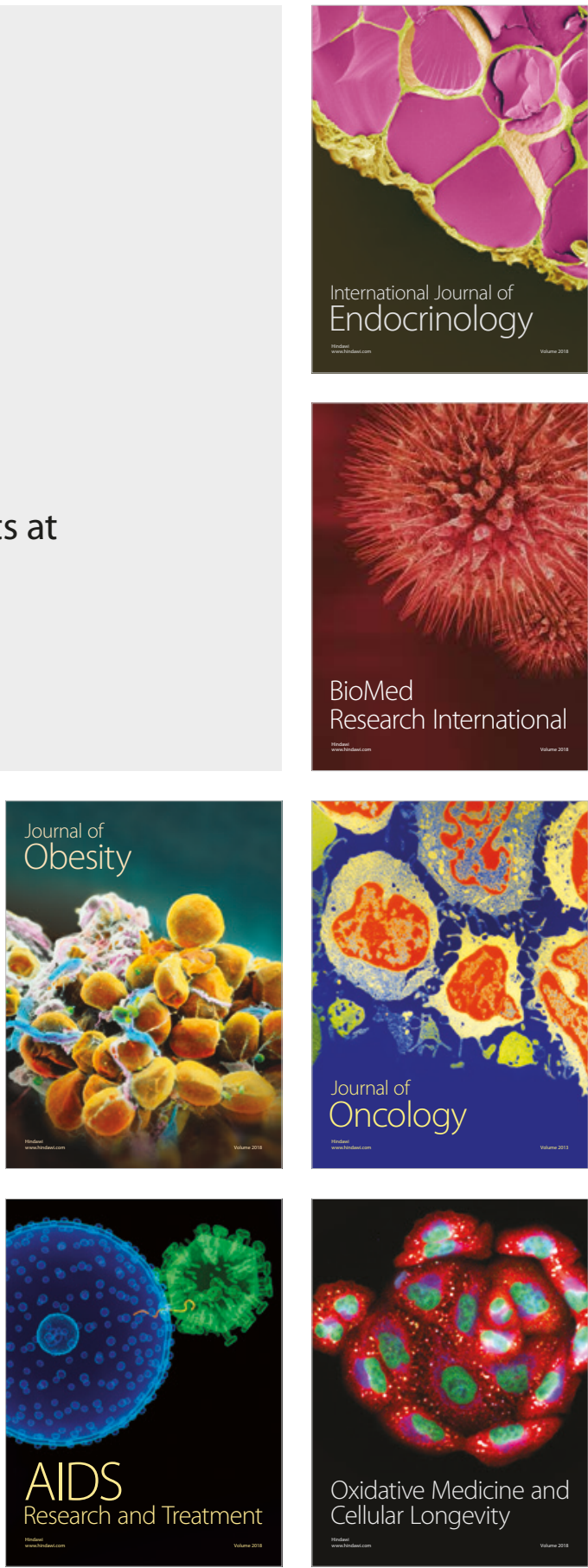